Research Article

\title{
Bifurcation Analysis for a Simple Dual-Rotor System with Nonlinear Intershaft Bearing Based on the Singularity Method
}

\author{
Peng Gao $(\mathbb{D})$, Yushu Chen, and Lei Hou \\ School of Astronautics, Harbin Institute of Technology, Harbin 150001, China \\ Correspondence should be addressed to Lei Hou; houlei@hit.edu.cn
}

Received 29 October 2019; Revised 18 January 2020; Accepted 8 May 2020; Published 20 May 2020

Academic Editor: Dario Di Maio

Copyright ( $\odot 2020$ Peng Gao et al. This is an open access article distributed under the Creative Commons Attribution License, which permits unrestricted use, distribution, and reproduction in any medium, provided the original work is properly cited.

\begin{abstract}
This paper aims to classify bifurcation modes for two interrelated primary resonances of a simple dual-rotor system under double frequency excitations. The four degree-of-freedom (4DOF) dynamic equations of the system considering the nonlinearity of the intershaft bearing can be obtained by using the assumed mode method (AMM) and Lagrange's equation. A simplified method for dynamic equations is developed due to the symmetry of rotors, based on which the amplitude frequency equations for two interrelated primary resonances are obtained by using the multiple scales method. Furthermore, the validity of the simplified method for dynamic equations and the amplitude frequency equations solved by the multiple scales method are confirmed by numerical verification. Afterwards, the bifurcation analysis for two interrelated primary resonances is carried out according to the two-state-variable singularity method. There exist a total of three different types of bifurcation modes because of double frequency excitations of the dual-rotor system and the nonlinearity of the intershaft bearing. The second primary resonance is more prone to have nonlinear dynamic characteristics than the first primary resonance. This discovery indicates that two interrelated primary resonances of the dual-rotor system may have different bifurcation modes under the same dynamic parameters.
\end{abstract}

\section{Introduction}

With the development of aero-engines towards higher thrust-weight ratio and better aerodynamic stability [1], the dual-rotor structure has been widely used in the field of aero-engines. As a vital part between the lower pressure (LP) rotor and the higher pressure (HP) rotor in the dualrotor system especially in aero-engines of fighters, the nonlinearity of the intershaft bearing [2] has achieved considerable attention [3]. There exist double frequency excitations, i.e., unbalanced excitations of LP and HP rotors, in the dual-rotor system due to the complexity of its structure. Moreover, under the nonlinear influence of the intershaft bearing, the dynamic behaviors of dual-rotor system will become much more complex and difficult to predict. Therefore, to clarify bifurcation modes of the dualrotor system under different dynamic parameters is very contributive to avoid some unexpected nonlinear dynamic behaviors $[4,5]$, so as to improve the operation safety of aero-engines.
There indeed exists some literature about the dynamic behaviors of the dual-rotor system in recent decades. Hibner [6] proposed a unique method, namely, the transfer-matrix method, to estimate vibratory responses of a dual-rotor system with the nonlinear damping coefficient in an aeroengine. Gupta et al. [7] developed a dual-rotor test rig to simulate the two spool aero-engine dynamically and compared experimental results and theoretical results for unbalance responses of the dual-rotor system. Ferraris et al. [8] presented a simple but realistic nonsymmetric coaxial dualrotor system and focused on the dynamic behaviors of the dual-rotor system under corotation and counterrotation. Different from Gupta et al. [7], a dual-rotor test rig composed of two coaxial shafts was developed by Guskov et al. [9], where two shafts are linked by an intershaft bearing and rotate individually. Zhang et al. $[10,11]$ successively put forward the whole-beat correlation method and the nonwhole beat correlation method to identify unbalance responses of a dual-rotor system with a slight rotation speed difference. Yang et al. [12] established a dual-rotor system 
with imbalance-rubbing coupled fault to study dynamic responses of aero-engines with fixed-point rubbing. Wang et al. $[13,14]$ also concentrated on the dynamic responses a dual-rotor structure of a whole aero-engine with bladecasing rubbing. Sun et al. $[15,16]$ investigated the nonlinear dynamical behaviors of a dual-rotor aero-engine with rub impact by means of an analytic method, namely, MHB-AFT (multiharmonic balance-alternating frequency/time domain). Nevertheless, the nonlinearity of the intershaft bearing is not considered in these dual-rotor models, and none of the above literature have classified bifurcation modes of the dual-rotor system.

The singularity theory is a mathematical method to study the morphology and classification of differentiable mapping near singular points [17]. Until 1985, Golubitsky and Schaeffer [18] elaborated the mathematical deductions and applications about the singularity theory in their books, based on which the singularity theory became a powerful mathematical tool to investigate bifurcation modes of the nonlinear dynamic system. Chen et al. $[19,20]$ applied the steady-state bifurcation theory of the dynamic system to investigate topological structures of bifurcation modes for a nonlinear dynamic system, and they proposed a new method, namely, C-L method, to study bifurcation modes for the nonlinear dynamic system of a single bifurcation parameter. Furthermore, Qin et al. [21, 22] developed the single state-variable singularity method into the two-state-variable singularity method through rigorous mathematical proof and gave mathematical formulas of the transition sets, i.e., the bifurcation set, the hysteresis set, and the double limit set. This research applied the two-state-variable singularity method to investigate bifurcation modes for the nonlinear dual-rotor system.

The purpose of this paper is to investigate bifurcation modes for two interrelated primary resonances of a dual-rotor system based on the two-state-variable singularity method, where the double frequency excitations of the dual-rotor system and the nonlinearity of the intershaft bearing are taken into consideration. A simplified method for dynamic equations is developed due to the symmetry of rotors, and the bifurcation parameters of the dual-rotor system are also reduced because of the amplitude frequency equations solved by the multiple scales method. Afterwards, the numerical integral is performed to confirm the validity of the simplified method and the amplitude frequency equations. Furthermore, the bifurcation analysis for two interrelated primary resonances of the dual-rotor system could be carried out based on the twostate-variable singularity method. It shows the second primary resonance is more prone to have nonlinear dynamic characteristics than the first primary resonance.

\section{Dynamic Equations of the Dual-Rotor System}

2.1. Dual-Rotor System. A simple two-disk dual-rotor system supported by four points with an intershaft bearing $[15,23]$ is depicted in Figure 1. The system is consisted of one LP rotor, one HP rotor, and an intershaft bearing. The LP rotor comprises a flexible shaft and a disk, while the HP rotor comprises a rigid rotor and a disk. The intershaft bearing, located between the LP rotor and HP rotor, is considered as a nonlinear elastic

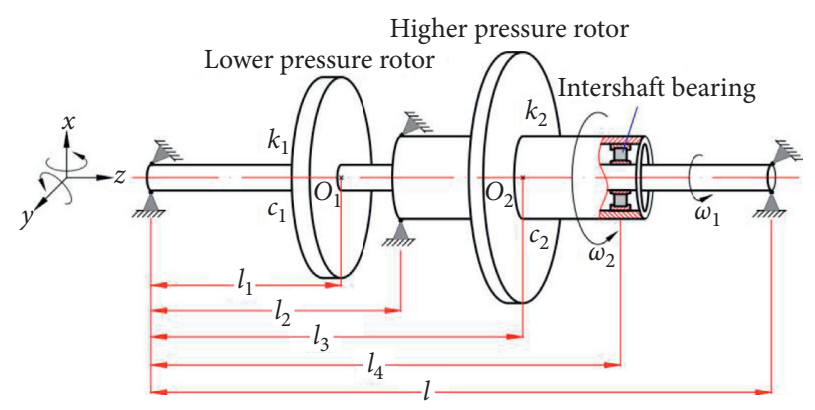

FIgURE 1: A simple two-disk dual-rotor system supported by four points with an intershaft bearing.

spring, which is the nonlinear source of the dual-rotor system. Herein, the subscript 1 denotes the LP rotor and the subscript 2 denotes the HP rotor without special instructions; $k, c, \omega$, and $O$ are the stiffness coefficient, the damping coefficient of the shaft, the rotation speed of the rotor, and the geometric center of the disk; $l_{i}(i=1 \sim 4)$ and $l$ are the lengths of shafts.

Figure 2 illustrates the lateral vibration of the dual-rotor system. The LP rotor is assumed as a flexible rotor while the HP rotor is assumed as a rigid rotor [24], since the LP shaft is much more slender than the HP shaft. Herein, $x_{1}$ and $y_{1}$ represent vertical and horizontal displacements of the LP disk; $x_{2}$ and $y_{2}$ represent vertical and horizontal displacements of the HP disk.

The LP shaft and HP shaft are considered as the Euler-Bernoulli beam, in which the deformations of shear and torsion are ignored. According to the assumed mode method [25], the LP shaft is a flexible shaft; thus, the modal shape function is assumed as a sine function, as

$$
f_{1}\left(z_{1}\right)=\sin \left(\frac{\pi z_{1}}{l}\right), \quad\left(z_{1} \in[0, l]\right)
$$

At any portion $z_{1}$, the vertical displacement $u_{1}$ and horizontal displacement $v_{1}$ of the LP shaft can be represented by vertical displacement $x_{1}$ and horizontal displacement $y_{1}$ of the LP disk due to the modal shape function equation (1), as

$$
\left\{\begin{array}{l}
u_{1}\left(z_{1}, t\right)=\frac{f_{1}\left(z_{1}\right)}{f_{1}\left(l_{1}\right)} x_{1}(t)=\frac{f_{1}\left(z_{1}\right)}{f_{1}\left(l_{1}\right)} x_{1}, \\
v_{1}\left(z_{1}, t\right)=\frac{f_{1}\left(z_{1}\right)}{f_{1}\left(l_{1}\right)} y_{1}(t)=\frac{f_{1}\left(z_{1}\right)}{f_{1}\left(l_{1}\right)} y_{1} .
\end{array}\right.
$$

The rotational angles of the LP shaft around $x$-axis $\theta_{x}$ and $y$-axis $\theta_{y}$ are both very small, and thus, they can also be represented by $x_{1}$ and $y_{1}$, as

$$
\left\{\begin{array}{l}
\theta_{x}\left(z_{1}, t\right) \approx-\frac{\partial v_{1}\left(z_{1}, t\right)}{\partial z_{1}}=-\frac{\mathrm{d} f_{1}\left(z_{1}\right)}{f_{1}\left(l_{1}\right) \mathrm{d} z_{1}} y_{1}(t)=-h_{1}\left(z_{1}\right) y_{1}, \\
\theta_{y}\left(z_{1}, t\right) \approx \frac{\partial u_{1}\left(z_{1}, t\right)}{\partial z_{1}}=\frac{\mathrm{d} f_{1}\left(z_{1}\right)}{f_{1}\left(l_{1}\right) \mathrm{d} z_{1}} x_{1}(t)=h_{1}\left(z_{1}\right) x_{1},
\end{array}\right.
$$

where $h_{1}\left(z_{1}\right)=\left(\mathrm{d} f_{1}\left(z_{1}\right) / f_{1}\left(l_{1}\right) \mathrm{d} z_{1}\right)$. 


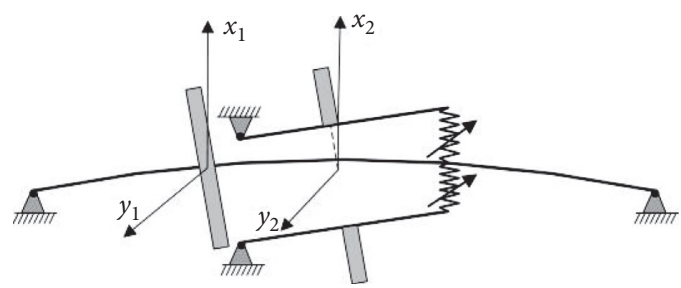

FIgURE 2: Lateral vibration of the dual-rotor system.

The LP disk is fixed with the LP shaft at $z_{1}=l_{1}$; thus, displacements and rotational angles of LP disk are just equal to displacements and rotational angles of LP shaft at $z_{1}=l_{1}$.
The kinetic energy of the LP rotor contains two parts, one is the kinetic energy of the LP disk $T_{d 1}$ and the other is the kinetic energy of the LP shaft $T_{s 1}$, as

$$
\begin{aligned}
T_{d 1} & =\frac{1}{2} m_{1}\left[\dot{u}_{1}^{2}\left(l_{1}\right)+\dot{v}_{1}^{2}\left(l_{1}\right)\right]+\frac{1}{2} J_{d 1}\left[\dot{\theta}_{x}^{2}\left(l_{1}\right)+\dot{\theta}_{y}^{2}\left(l_{1}\right)\right]+\frac{1}{2} J_{p 1}\left[\omega_{1}^{2}-2 \omega_{1} \theta_{x}\left(l_{1}\right) \dot{\theta}_{y}\left(l_{1}\right)\right] \\
& =\frac{1}{2} m_{1}\left(\dot{x}_{1}^{2}+\dot{y}_{1}^{2}\right)+\frac{1}{2} J_{d 1} h_{1}^{2}\left(l_{1}\right)\left(\dot{x}_{1}^{2}+\dot{y}_{1}^{2}\right)+\frac{1}{2} J_{p 1}\left(\omega_{1}^{2}+2 \omega_{1} h_{1}^{2}\left(l_{1}\right) \dot{x}_{1} y_{1}\right), \\
T_{s 1} & =\frac{\rho_{1} \pi r_{1}^{2}}{2} \int_{0}^{l}\left(\dot{u}_{1}^{2}+\dot{v}_{1}^{2}\right) \mathrm{d} z_{1}+\frac{\rho_{1} I_{1}}{2} \int_{0}^{l}\left(\dot{\theta}_{x}^{2}+\dot{\theta}_{y}^{2}\right) \mathrm{d} z_{1}+\rho_{1} I_{1} \int_{0}^{l}\left(\omega_{1}^{2}-2 \omega_{1} \theta_{x} \dot{\theta}_{y}\right) \mathrm{d} z_{1} \\
& =\frac{\rho_{1} \pi r_{1}^{2}}{2 f_{1}^{2}\left(l_{1}\right)}\left(\dot{x}_{1}^{2}+\dot{y}_{1}^{2}\right) \int_{0}^{l} f_{1}^{2} \mathrm{~d} z_{1}+\frac{\rho_{1} I_{1}}{2}\left(\dot{x}_{1}^{2}+\dot{y}_{1}^{2}\right) \int_{0}^{l} h_{1}^{2} \mathrm{~d} z_{1}+\rho_{1} I_{1}\left(\omega_{1}^{2} l+2 \omega_{1} \dot{x}_{1} y_{1} \int_{0}^{l} h_{1}^{2} \mathrm{~d} z_{1}\right),
\end{aligned}
$$

where $m_{1}$ is the mass of the LP rotor; $J_{p 1}$ and $J_{d 1}$ are the LP disk's polar and diameter moment of inertia; $r_{1}$ and $\rho_{1}$ are the radius and the density of the LP shaft (solid shaft); and the LP shaft's area moment of inertia is $I_{1}=\left(\pi r_{1}^{4} / 4\right)$.

The HP shaft is a stubby shaft, which can be considered as a rigid rotor; thus, the modal shape function is

$$
f_{2}\left(z_{2}\right)=z_{2}-l_{2}, \quad z_{2} \in\left(l_{2}, l_{4}\right) .
$$

At any portion $z_{2}$, the vertical displacement $u_{2}$ and the horizontal displacement $v_{2}$ of the HP shaft can be represented by vertical displacement $x_{2}$ and horizontal displacement $y_{2}$ of the HP disk due to the modal shape function equation (5), as

$$
\left\{\begin{array}{l}
u_{2}\left(z_{2}, t\right)=\frac{f_{2}\left(z_{2}\right)}{f_{2}\left(l_{3}\right)} x_{2}(t)=\frac{f_{2}\left(z_{2}\right)}{f_{2}\left(l_{3}\right)} x_{2}, \\
v_{2}\left(z_{2}, t\right)=\frac{f_{2}\left(z_{2}\right)}{f_{2}\left(l_{3}\right)} y_{2}(t)=\frac{f_{2}\left(z_{2}\right)}{f_{2}\left(l_{3}\right)} y_{2} .
\end{array}\right.
$$

The rotational angles of HP shaft around $x$-axis $\varphi_{x}$ and $y$ axis $\varphi_{y}$ are both very small; thus, they can also be represented by $x_{2}$ and $y_{2}$, as

$$
\left\{\begin{array}{l}
\varphi_{x}\left(z_{2}, t\right) \approx-\frac{\partial v_{2}\left(z_{2}, t\right)}{\partial z_{2}}=-\frac{\mathrm{d} f_{2}\left(z_{2}\right)}{f_{2}\left(l_{3}\right) \mathrm{d} z_{2}} y_{2}(t)=-h_{2}\left(z_{2}\right) y_{2}, \\
\varphi_{y}\left(z_{2}, t\right) \approx \frac{\partial u_{2}\left(z_{2}, t\right)}{\partial z_{2}}=\frac{\mathrm{d} f_{2}\left(z_{2}\right)}{f_{2}\left(l_{3}\right) \mathrm{d} z_{2}} x_{2}(t)=h_{2}\left(z_{2}\right) x_{2},
\end{array}\right.
$$

where $h_{2}\left(z_{2}\right)=\left(\mathrm{d} f_{2}\left(z_{2}\right) / f_{2}\left(l_{3}\right) \mathrm{d} z_{2}\right)=\left(1 /\left(l_{3}-l_{2}\right)\right)$.

The HP disk is fixed with the HP shaft at $z_{2}=l_{3}$; thus, displacements and rotational angles of HP disk are just equal to displacements and rotational angles of HP shaft at $z_{2}=l_{3}$. The kinetic energy of the HP rotor contains two parts, one is the kinetic energy of the HP disk $T_{d 2}$ and the other is the kinetic energy of the HP shaft $T_{s 2}$, as

$$
\begin{aligned}
T_{d 2} & =\frac{1}{2} m_{2}\left[\dot{u}_{2}^{2}\left(l_{3}\right)+\dot{v}_{2}^{2}\left(l_{3}\right)\right]+\frac{1}{2} J_{d 2}\left(\dot{\varphi}_{x}^{2}\left(l_{3}\right)+\dot{\varphi}_{y}^{2}\left(l_{3}\right)\right)+\frac{1}{2} J_{p 2}\left(\omega_{2}^{2}-2 \omega_{2} \varphi_{x}\left(l_{3}\right) \dot{\varphi}_{y}\left(l_{3}\right)\right) \\
& =\frac{1}{2} m_{2}\left(\dot{x}_{2}^{2}+\dot{y}_{2}^{2}\right)+\frac{1}{2} J_{d 2} h_{2}^{2}\left(l_{3}\right)\left(\dot{x}_{2}^{2}+\dot{y}_{2}^{2}\right)+\frac{1}{2} J_{p 2}\left(\omega_{2}^{2}+2 \omega_{2} h_{2}^{2}\left(l_{3}\right) \dot{x}_{2} y_{2}\right), \\
T_{s 2} & =\frac{\rho_{2} \pi\left(r_{3}^{2}-r_{2}^{2}\right)}{2} \int_{l_{2}}^{l_{4}}\left(\dot{u}_{2}^{2}+\dot{v}_{2}^{2}\right) \mathrm{d} z_{2}+\frac{\rho_{2} I_{2}}{2} \int_{l_{2}}^{l_{4}}\left(\dot{\varphi}_{x}^{2}+\dot{\varphi}_{y}^{2}\right) \mathrm{d} z_{2}+\rho_{2} I_{2} \int_{l_{2}}^{l_{4}}\left(\omega_{2}^{2}-2 \omega_{2} \varphi_{x} \dot{\varphi}_{y}\right) \mathrm{d} z_{2} \\
& =\frac{\rho_{2} \pi\left(r_{3}^{2}-r_{2}^{2}\right)}{2 f_{2}^{2}\left(l_{3}\right)}\left(\dot{x}_{2}^{2}+\dot{y}_{2}^{2}\right) \int_{l_{2}}^{l_{4}} f_{2}^{2} \mathrm{~d} z_{2}+\frac{\rho_{2} I_{2}}{2}\left(\dot{x}_{2}^{2}+\dot{y}_{2}^{2}\right) \int_{l_{2}}^{l_{4}} h_{2}^{2} \mathrm{~d} z_{2}+\rho_{2} I_{2}\left[\omega_{2}^{2}\left(l_{4}-l_{2}\right)+2 \omega_{2} \dot{x}_{2} y_{2} \int_{l_{2}}^{l_{4}} h_{2}^{2} \mathrm{~d} z_{2}\right],
\end{aligned}
$$


where $m_{2}$ is the mass of the HP rotor; $J_{p 2}$ and $J_{d 2}$ are the HP disk's polar and diameter moment of inertia; and $r_{2}, r_{3}$, and $\rho_{2}$ are the inner and the outer radiuses, and the density of the HP shaft (hollow shaft); the HP shaft's area moment of inertia is $I_{2}=\left(\pi\left(r_{3}^{4}-r_{2}^{4}\right) / 4\right)$.

Thus, the kinetic energy of the system can be obtained as

$$
T=T_{d 1}+T_{s 1}+T_{d 2}+T_{s 2} \text {. }
$$

The potential energy of the system is

$$
V=\frac{1}{2} k_{1}\left(x_{1}^{2}+y_{1}^{2}\right)+\frac{1}{2} k_{2}\left(x_{2}^{2}+y_{2}^{2}\right)
$$

where $k_{1}$ and $k_{2}$ are the stiffness coefficients of LP shaft and HP shaft.

Rayleigh's dissipation energy of the system is

$$
D=\frac{1}{2} c_{1}\left(\dot{x}_{1}^{2}+\dot{y}_{1}^{2}\right)+\frac{1}{2} c_{2}\left(\dot{x}_{2}^{2}+\dot{y}_{2}^{2}\right)
$$

where $c_{1}$ and $c_{2}$ are the damping coefficients of LP shaft and HP shaft.

The generalized force virtual work of the system is

$$
\begin{aligned}
\delta W= & m_{1} \omega_{1}^{2} e_{1} \cos \left(\omega_{1} t\right) \delta x_{1}+m_{1} \omega_{1}^{2} e_{1} \sin \left(\omega_{1} t\right) \delta y_{1} \\
& +m_{2} \omega_{2}^{2} e_{2} \cos \left(\omega_{2} t\right) \delta x_{2}+m_{2} \omega_{2}^{2} e_{2} \sin \left(\omega_{2} t\right) \delta y_{2} \\
& -F_{x}\left(F_{1} \delta x_{1}-F_{2} \delta x_{2}\right)-F_{y}\left(F_{1} \delta y_{1}-F_{2} \delta y_{2}\right),
\end{aligned}
$$

where $F_{1}=\left(f_{1}\left(l_{4}\right) / f_{1}\left(l_{1}\right)\right)$ and $F_{2}=\left(f_{2}\left(l_{4}\right) / f_{2}\left(l_{3}\right)\right) ; F_{x}$ and $F_{y}$ represent vertical and horizontal nonlinear restoring forces of the intershaft bearing; $e_{1}$ and $e_{2}$ represent unbalances of LP and HP rotors; and $\delta \bullet\left(x_{1}, y_{1}, x_{2}, y_{2}\right)$ represents the corresponding virtual displacement in the corresponding generalized coordinate.

2.2. Nonlinear Restoring Forces of the Intershaft Bearing. The inner race and the LP rotor are interference fit at $z_{1}=l_{4}$; thus, vertical displacement $x_{\mathrm{i}}$ and horizontal displacement $y_{\mathrm{i}}$ of the inner race are just equal to vertical displacement and horizontal displacement of the LP shaft at $z_{1}=l_{4}$; substituting $z_{1}=l_{4}$ into equation (2), we obtain

$$
\left\{\begin{array}{l}
x_{i}=u_{1}\left(l_{4}, t\right)=\frac{f_{1}\left(l_{4}\right)}{f_{1}\left(l_{1}\right)} x_{1}=F_{1} x_{1}, \\
y_{i}=v_{1}\left(l_{4}, t\right)=\frac{f_{1}\left(l_{4}\right)}{f_{1}\left(l_{1}\right)} y_{1}=F_{1} y_{1} .
\end{array}\right.
$$

The outer race and the HP rotor are interference fit at $z_{2}=l_{4}$; thus, vertical displacement $x_{o}$ and horizontal displacement $y_{o}$ of the outer race are just equal to vertical displacement and horizontal displacement of the HP shaft at $z_{2}=l_{4}$; substituting $z_{2}=l_{4}$ into equation (6), we obtain

$$
\left\{\begin{array}{l}
x_{o}=u_{2}\left(l_{4}, t\right)=\frac{f_{2}\left(l_{4}\right)}{f_{2}\left(l_{1}\right)} x_{2}=F_{2} x_{2} \\
y_{o}=v_{2}\left(l_{4}, t\right)=\frac{f_{2}\left(l_{4}\right)}{f_{2}\left(l_{1}\right)} y_{2}=F_{2} y_{2}
\end{array}\right.
$$

The intershaft bearing can be seen as a nonlinear elastic spring $[26,27]$, which is consisted of a linear stiffness $k_{s}$ and a cubic nonlinear stiffness $k_{n}$. Therefore, vertical and horizontal nonlinear restoring forces of the intershaft bearing are

$$
\begin{aligned}
F_{x} & =k_{s}\left(x_{i}-x_{o}\right)+k_{n}\left(x_{i}-x_{o}\right)\left[\left(x_{i}-x_{o}\right)^{2}-3\left(y_{i}-y_{o}\right)^{2}\right] \\
& =k_{s}\left(F_{1} x_{1}-F_{2} x_{2}\right)+k_{n}\left(F_{1} x_{1}-F_{2} x_{2}\right)\left[\left(F_{1} x_{1}-F_{2} x_{2}\right)^{2}-3\left(F_{1} y_{1}-F_{2} y_{2}\right)^{2}\right], \\
F_{y} & =k_{s}\left(y_{i}-y_{o}\right)+k_{n}\left(y_{i}-y_{o}\right)\left[\left(y_{i}-y_{o}\right)^{2}-3\left(x_{i}-x_{o}\right)^{2}\right] \\
& =k_{s}\left(F_{1} y_{1}-F_{2} y_{2}\right)+k_{n}\left(F_{1} y_{1}-F_{2} y_{2}\right)\left[\left(F_{1} y_{1}-F_{2} y_{2}\right)^{2}-3\left(F_{1} x_{1}-F_{2} x_{2}\right)^{2}\right],
\end{aligned}
$$

where $k_{s}$ denotes the linear stiffness of the intershaft bearing while $k_{n}$ denotes the cubic nonlinear stiffness of the intershaft bearing.

Comparing equations (15a) and (15b) with conventional cubic nonlinear elastic spring, the symmetry of the intershaft bearing in vertical and horizontal directions is taken into consideration for the nonlinear restoring forces of the intershaft bearing.

2.3. Dynamic Equations of the Dual-Rotor System. The 4DOF dynamic equations of the dual-rotor system can be obtained with the aid of Lagrange's equation of the second kind, as

$$
M_{1} \ddot{x}_{1}+c_{1} \dot{x}_{1}+\omega_{1} g_{1} \dot{y}_{1}+k_{1} x_{1}=m_{1} \omega_{1}^{2} e_{1} \cos \omega_{1} t-F_{1} F_{x}
$$

$$
M_{1} \ddot{y}_{1}+c_{1} \dot{y}_{1}-\omega_{1} g_{1} \dot{x}_{1}+k_{1} y_{1}=m_{1} \omega_{1}^{2} e_{1} \sin \omega_{1} t-F_{1} F_{y},
$$

$$
M_{2} \ddot{x}_{2}+c_{2} \dot{x}_{2}+\omega_{2} g_{2} \dot{y}_{2}+k_{2} x_{2}=m_{2} \omega_{2}^{2} e_{2} \cos \omega_{2} t+F_{2} F_{x}
$$

$$
M_{2} \ddot{y}_{2}+c_{2} \dot{y}_{2}-\omega_{2} g_{2} \dot{x}_{2}+k_{2} y_{2}=m_{2} \omega_{2}^{2} e_{2} \sin \omega_{2} t+F_{2} F_{y},
$$


where $\quad M_{1}=m_{1}+H_{1}^{2} J_{d 1}+\left(\rho_{1} \pi r_{1}^{2} / f_{1}^{2}\left(l_{1}\right)\right) \int_{0}^{l} f_{1}^{2} \mathrm{~d} z_{1}+\rho_{1} I_{1}$ $\int_{0}^{l} h_{1}^{2} \mathrm{~d} z_{1}, M_{2}=m_{2}+H_{2}^{2} J_{d 2}+\left(\rho_{2} \pi\left(r_{3}^{2}-r_{2}^{2}\right) / f_{2}^{2}\left(l_{3}\right)\right) \int_{l_{2}}^{l_{4}} f_{2}^{2} \mathrm{~d} z_{2}+$ $\rho_{2} I_{2} \int_{l_{2}}^{l_{4}} h_{2}^{2} \mathrm{~d} z_{2}, g_{1}=H_{1}^{2} J_{p 1}+2 \rho_{1} I_{1} \int_{0}^{l} h_{1}^{2} \mathrm{~d} z_{1}$, and $g_{2}=H_{2}^{2} J_{p 2}+2$ $\rho_{2} I_{2} \int_{l_{2}}^{l_{4}} h_{2}^{2} \mathrm{~d} z_{2}$. Moreover, the gyroscopic effect is reflected in the parameters $M_{1}, M_{2}, g_{1}$, and $g_{2}$.

The dimensionless dynamic equations of the dual-rotor system are

$$
\begin{gathered}
X_{1}^{\prime \prime}+C_{1} X_{1}^{\prime}+G_{1} Y_{1}^{\prime}+\frac{k_{1}}{M_{1} \omega_{1}^{2}} X_{1}=E_{1} \cos \tau-\frac{F_{1}}{M_{1} \omega_{1}^{2}} F_{X}, \\
Y_{1}^{\prime \prime}+C_{1} Y_{1}^{\prime}-G_{1} X_{1}^{\prime}+\frac{k_{1}}{M_{1} \omega_{1}^{2}} Y_{1}=E_{1} \sin \tau-\frac{F_{1}}{M_{1} \omega_{1}^{2}} F_{Y},
\end{gathered}
$$

$X_{2}^{\prime \prime}+C_{2} X_{2}^{\prime}+G_{2} Y_{2}^{\prime}+\frac{k_{2}}{M_{2} \omega_{1}^{2}} X_{2}=\lambda^{2} E_{2} \cos (\lambda \tau)+\frac{F_{2}}{M_{2} \omega_{1}^{2}} F_{X}$

$$
Y_{2}^{\prime \prime}+C_{2} Y_{2}^{\prime}-G_{2} X_{2}^{\prime}+\frac{k_{2}}{M_{2} \omega_{1}^{2}} Y_{2}=\lambda^{2} E_{2} \sin (\lambda \tau)+\frac{F_{2}}{M_{2} \omega_{1}^{2}} F_{Y},
$$

where $\tau=\omega_{1} t, \quad \lambda=\left(\omega_{2} / \omega_{1}\right) \quad$ (rotation speed ratio), $E_{1}=\left(m_{1} / M_{1}\right), \quad E_{2}=\left(m_{2} / M_{2}\right) \cdot\left(e_{2} / e_{1}\right), \quad X_{1}=\left(x_{1} / e_{1}\right)$, $Y_{1}=\left(y_{1} / e_{1}\right), X_{2}=\left(x_{2} / e_{1}\right), Y_{2}=\left(y_{2} / e_{1}\right), C_{1}=\left(c_{1} / M_{1} \omega_{1}\right)$, $C_{2}=\left(c_{2} / M_{2} \omega_{1}\right), G_{1}=\left(g_{1} / M_{1}\right)$, and $G_{2}=\left(\lambda g_{2} / M_{2}\right)$ are the dimensionless parameters.

The dimensionless nonlinear restoring force of the intershaft bearing is

$$
\begin{aligned}
{\left[\begin{array}{c}
F_{X} \\
F_{Y}
\end{array}\right] } & =\left[\begin{array}{ll}
\frac{F_{x}}{e_{1}} & \frac{F_{y}}{e_{1}}
\end{array}\right]^{T}=\left[\begin{array}{l}
k_{s}\left(X_{i}-X_{o}\right)+e_{1}^{2} k_{n}\left(X_{i}-X_{o}\right)\left[\left(X_{i}-X_{o}\right)^{2}-3\left(Y_{i}-Y_{o}\right)^{2}\right] \\
k_{s}\left(Y_{i}-Y_{o}\right)+e_{1}^{2} k_{n}\left(Y_{i}-Y_{o}\right)\left[\left(Y_{i}-Y_{o}\right)^{2}-3\left(X_{i}-X_{o}\right)^{2}\right]
\end{array}\right] \\
& =\left[\begin{array}{l}
k_{s}\left(F_{1} X_{1}-F_{2} X_{2}\right)+e_{1}^{2} k_{n}\left(F_{1} X_{1}-F_{2} X_{2}\right)\left[\left(F_{1} X_{1}-F_{2} X_{2}\right)^{2}-3\left(F_{1} Y_{1}-F_{2} Y_{2}\right)^{2}\right] \\
k_{s}\left(F_{1} Y_{1}-F_{2} Y_{2}\right)+e_{1}^{2} k_{n}\left(F_{1} Y_{1}-F_{2} Y_{2}\right)\left[\left(F_{1} Y_{1}-F_{2} Y_{2}\right)^{2}-3\left(F_{1} X_{1}-F_{2} X_{2}\right)^{2}\right]
\end{array}\right] .
\end{aligned}
$$

The parameters of the dual-rotor system [23] are shown as follows:

$$
\begin{aligned}
m_{1} & =97.37 \mathrm{~kg}, \\
J_{p 1} & =3.6907 \mathrm{~kg} \cdot \mathrm{m}^{2}, \\
J_{d 1} & =1.8454 \mathrm{~kg} \cdot \mathrm{m}^{2}, \\
m_{2} & =108.3 \mathrm{~kg}, \\
J_{p 2} & =4.0114 \mathrm{~kg} \cdot \mathrm{m}^{2}, \\
J_{d 2} & =2.0060 \mathrm{~kg} \cdot \mathrm{m}^{2}, \\
k_{1} & =k_{2}=8 \times 10^{7} \mathrm{~N} / \mathrm{m}, \\
c_{1} & =c_{2}=600 \mathrm{~N} \cdot \mathrm{s} / \mathrm{m}, \\
\rho_{1} & =\rho_{2}=7800 \mathrm{~kg} / \mathrm{m}^{3}, \\
e_{1} & =1 \mu \mathrm{m}, \\
e_{2} & =3 \mu \mathrm{m}, \\
l & =1.5913 \mathrm{~m}, \\
l_{1} & =0.2250 \mathrm{~m}, \\
l_{2} & =0.3555 \mathrm{~m}, \\
l_{3} & =0.8675 \mathrm{~m}, \\
l_{4} & =1.4918 \mathrm{~m}, \\
r_{1} & =20 \mathrm{~mm}, \\
r_{2} & =30 \mathrm{~mm}, \\
r_{3} & =35 \mathrm{~mm}, \\
\lambda & =1.2, \\
k_{s} & =3 \times 10^{8} \mathrm{~N} / \mathrm{m}, \\
k_{n} & =1 \times 10^{23} \mathrm{~N} / \mathrm{m}^{3} .
\end{aligned}
$$

\section{Multiple Scales Solution}

3.1. Dynamic Equations Transferring. Considering the symmetry of LP and HP rotors in vertical and horizontal directions, the 4 DOF equations $(17 \mathrm{a})-(17 \mathrm{~d})$ can be simplified into a $2 \mathrm{DOF}$ equation with the help of a complexnumber coordinate [28]. Let $r_{1}=X_{1}+i Y_{1}, r_{2}=X_{2}+i Y_{2}$, and $F_{\mathrm{b}}=F_{X}+i F_{Y} \quad\left(i^{2}=-1\right)$; equations $(17 \mathrm{a})-(17 \mathrm{~d})$ are transformed into

$$
\begin{aligned}
& r_{1}^{\prime \prime}+\left(C_{1}-i G_{1}\right) r_{1}^{\prime}+\frac{k_{1}}{M_{1} \omega_{1}^{2}} r_{1}=E_{1} e^{i \tau}-\frac{F_{1}}{M_{1} \omega_{1}^{2}} F_{b}, \\
& r_{2}^{\prime \prime}+\left(C_{2}-i G_{2}\right) r_{2}^{\prime}+\frac{k_{2}}{M_{2} \omega_{1}^{2}} r_{2}=\lambda^{2} E_{2} e^{i \lambda \tau}+\frac{F_{2}}{M_{2} \omega_{1}^{2}} F_{b} .
\end{aligned}
$$

The nonlinear force of the intershaft bearing in the complex-number coordinate (deduction process in Appendix) is

$$
\begin{aligned}
F_{b} & =F_{X}+i F_{Y}=k_{s}\left(r_{i}-r_{o}\right)+e_{1}^{2} k_{n}\left(\bar{r}_{i}-\bar{r}_{o}\right)^{3} \\
& =k_{s}\left(F_{1} r_{1}-F_{2} r_{2}\right)+e_{1}^{2} k_{n}\left(F_{1} \bar{r}_{1}-F_{2} \bar{r}_{2}\right)^{3} .
\end{aligned}
$$

Substitute the nonlinear force equation (21) into equations (20a) and (20b) and transpose terms as 


$$
\begin{aligned}
r_{1}^{\prime \prime}+\frac{k_{1}+F_{1}^{2} k_{s}}{M_{1} \omega_{1}^{2}} r_{1}-\frac{F_{1} F_{2} k_{s}}{M_{1} \omega_{1}^{2}} r_{2}= & E_{1} e^{i \tau}-\left(C_{1}-i G_{1}\right) r_{1}^{\prime} \\
& -\frac{F_{1} e_{1}^{2} k_{n}}{M_{1} \omega_{1}^{2}}\left(F_{1} \bar{r}_{1}-F_{2} \bar{r}_{2}\right)^{3},
\end{aligned}
$$

$$
\begin{aligned}
r_{2}^{\prime \prime}-\frac{F_{1} F_{2} k_{s}}{M_{2} \omega_{1}^{2}} r_{1}+\frac{k_{2}+F_{2}^{2} k_{s}}{M_{2} \omega_{1}^{2}} r_{2}= & \lambda^{2} E_{2} e^{i \lambda \tau}-\left(C_{2}-i G_{2}\right) r_{2}^{\prime} \\
& +\frac{F_{2} e_{1}^{2} k_{n}}{M_{2} \omega_{1}^{2}}\left(F_{1} \bar{r}_{1}-F_{2} \bar{r}_{2}\right)^{3}
\end{aligned}
$$

Equations (22a) and (22b) in a matrix form is

$$
\begin{aligned}
& \mathbf{r}^{\prime \prime}+\mathbf{K r}=\mathbf{Q}, \\
& \text { where } \mathbf{r}=\left[\begin{array}{l}
r_{1} \\
r_{2}
\end{array}\right], \\
& \left(1 / \omega_{1}^{2}\right)\left[\begin{array}{cc}
\left(k_{1}+F_{1}^{2} k_{s}\right) / M_{1} & -\left(F_{1} F_{2} k_{s} / M_{1}\right) \\
-\left(F_{1} F_{2} k_{s} / M_{2}\right) & \left(k_{2}+F_{2}^{2} k_{s}\right) / M_{2}
\end{array}\right], \\
& \mathbf{Q}=\left[\begin{array}{l}
Q_{1} \\
Q_{2}
\end{array}\right]=\quad\left[E_{1} e^{i \tau}-\left(C_{1}-i G_{1}\right) r_{1}^{\prime}-\left(F_{1} e_{1}^{2} k_{n} / M_{1} \omega_{1}^{2}\right)\right. \\
& \left(F_{1} \bar{r}_{1}-F_{2} \bar{r}_{2}\right)^{3} \lambda^{2} E_{2} e^{i \lambda \tau}-\left(C_{2}-i G_{2}\right) r_{2}^{\prime}+\left(F_{2} e_{1}^{2} k_{n} / M_{2} \omega_{1}^{2}\right) \\
& \left.\left(F_{1} \bar{r}_{1}-F_{2} \bar{r}_{2}\right)^{3}\right] .
\end{aligned}
$$

Eigenvectors and eigenvalues of the matrix $\mathbf{K}$ are

$$
(\boldsymbol{\Phi}, \mathbf{\Omega})=\operatorname{eig}(\mathbf{K})
$$

where eig $(\cdot)$ is a function to compute eigenvectors and ei-

genvalues of the matrix.
Let eigenvalues be $\Omega=\left(1 / \omega_{1}^{2}\right)\left[\begin{array}{cc}\Omega_{1}^{2} & 0 \\ 0 & \Omega_{2}^{2}\end{array}\right]$, where $\Omega_{1}$ and $\Omega_{2}$ are the first- and second-order critical speedof the system. And let eigenvectors be $\Phi=\left[\begin{array}{cc}p_{1} & p_{2} \\ 1 & 1\end{array}\right]$; the inverse matrix of eigenvectors is $\Phi^{-1}=\left[\begin{array}{cc}q_{1} & 1-q_{2} \\ -q_{1} & q_{2}\end{array}\right]$, where $q_{1}=$ $\left(1 /\left(p_{1}-p_{2}\right)\right)$ and $q_{2}=\left(p_{1} /\left(p_{1}-p_{2}\right)\right)$.

Let both sides of equation (23) be premultiplied by $\Phi^{-1}$ and then expanded into a $2 \mathrm{DoF}$ equation as

$$
\begin{aligned}
& R_{1}^{\prime \prime}+\frac{\Omega_{1}^{2}}{\omega_{1}^{2}} R_{1}=q_{1} Q_{1}+\left(1-q_{2}\right) Q_{2}, \\
& R_{2}^{\prime \prime}+\frac{\Omega_{2}^{2}}{\omega_{1}^{2}} R_{2}=-q_{1} Q_{1}+q_{2} Q_{2},
\end{aligned}
$$

where $R_{1}=q_{1} r_{1}+\left(1-q_{2}\right) r_{2}$ and $R_{2}=-q_{1} r_{1}+q_{2} r_{2}$.

There are some assumptions as follows:

(1) The damping of the system is weak damping, and the nonlinearity of the system is weak nonlinearity

(2) $\Omega_{2} \gg \Omega_{1} \quad\left(\Omega_{2}=\eta \Omega_{1}, \eta \approx 5.10\right)$, i.e., second-order critical speed is far greater than first-order critical speed. This paper focuses on primary resonances, i.e., first-order critical speed
The dual-rotor system is excited by double frequency excitations, i.e., the unbalances of LP rotor and HP rotor. Therefore, first-order critical speed contains two interrelated primary resonances, one of which is excited by the unbalance of the HP rotor and the other is excited by the unbalance of the LP rotor [23]. When the dual-rotor system rotates at a constant rotation speed ratio $\lambda=\left(\omega_{2} / \omega_{1}\right)$, in general, $\lambda>1$ because the HP rotor rotates faster than the LP rotor. Thus, with the increase of the rotation speed, the first primary resonance is excited by the unbalance of the HP rotor at $\omega_{1} \approx\left(\Omega_{1} / \lambda\right)\left(\omega_{2} \approx \Omega_{1}\right)$ while the second primary resonance is excited by the unbalance of the LP rotor at $\omega_{1} \approx \Omega_{1}$.

3.2. First Primary Resonance at $\omega_{1} \approx\left(\Omega_{1} / \lambda\right)\left(\omega_{2} \approx \Omega_{1}\right)$. Time scales $T_{1}=\tau$ and $T_{2}=\varepsilon \tau$, and the tuning parameter $\sigma_{1}$ satisfies

$$
\frac{\Omega_{1}^{2}}{\omega_{1}^{2}}=\lambda^{2}-\varepsilon \sigma_{1}
$$

Assume that solutions of equations (25a) and (25b) are

$$
\begin{aligned}
& R_{1}=R_{11}+\varepsilon R_{12}, \\
& R_{2}=R_{21}+\varepsilon R_{22} .
\end{aligned}
$$

Substitute equations (26)-(27b) into equations (25a) and (25b) and equate coefficients of $\varepsilon^{0}$ and $\varepsilon^{1}$ to zero, as $\varepsilon^{0}$ is

$$
\begin{gathered}
D_{1}^{2} R_{11}+\lambda^{2} R_{11}=q_{1} E_{1} e^{i T_{1}}, \\
D_{1}^{2} R_{21}+(\lambda \eta)^{2} R_{21}=-q_{1} E_{1} e^{i T_{1}} .
\end{gathered}
$$

Also, $\varepsilon^{1}$ is

$$
\begin{aligned}
D_{1}^{2} R_{12}+\lambda^{2} R_{12}= & -2 D_{1} D_{2} R_{11}+\sigma_{1} R_{11}+\left(1-q_{2}\right) \lambda^{2} E_{2} e^{i \lambda T_{1}} \\
& -q_{1}\left(C_{1}-i G_{1}\right)\left(p_{1} D_{1} R_{11}+p_{2} D_{1} R_{21}\right) \\
& -\left(1-q_{2}\right)\left(C_{2}-i G_{2}\right)\left(D_{1} R_{11}+D_{1} R_{21}\right) \\
& +\gamma_{1}\left[\left(p_{1} F_{1}-F_{2}\right) \bar{R}_{11}+\left(p_{2} F_{1}-F_{2}\right) \bar{R}_{21}\right]^{3},
\end{aligned}
$$

$$
\begin{aligned}
D_{1}^{2} R_{22}+(\lambda \eta)^{2} R_{22}= & -2 D_{1} D_{2} R_{21}+\sigma_{1} \eta^{2} R_{21}-q_{2} \lambda^{2} E_{2} e^{i \lambda T_{1}} \\
& +q_{1}\left(C_{1}-i G_{1}\right)\left(p_{1} D_{1} R_{11}+p_{2} D_{1} R_{21}\right) \\
& -q_{2}\left(C_{2}-i G_{2}\right)\left(D_{1} R_{11}+D_{1} R_{21}\right) \\
& +\gamma_{2}\left[\left(p_{1} F_{1}-F_{2}\right) \bar{R}_{11}+\left(p_{2} F_{1}-F_{2}\right) \bar{R}_{21}\right]^{3}
\end{aligned}
$$

where $D_{1}=\left(\partial(\cdot) / \partial T_{1}\right), D_{2}=\left(\partial(\cdot) / \partial T_{2}\right), \gamma_{1}=\left[\left(\left(1-q_{2}\right) F_{2} /\right.\right.$ $\left.\left.M_{2} \omega_{1}^{2}\right)-\left(q_{1} F_{1} / M_{1} \omega_{1}^{2}\right)\right] e_{1}^{2} k_{n}, \quad \gamma_{2}=\left[\left(q_{2} F_{2} / M_{2} \omega_{1}^{2}\right)+\left(q_{1} F_{1} /\right.\right.$ $\left.\left.M_{1} \omega_{1}^{2}\right)\right] e_{1}^{2} k_{n}$, and $\eta=\left(\Omega_{2} / \Omega_{1}\right)$.

Assume that general solutions of equations (28a) and (28b) are

$$
\begin{aligned}
& R_{11}=A_{1} e^{i\left(\lambda T_{1}+\varphi_{1}\right)}+c c+a_{1} e^{i T_{1}}, \\
& R_{21}=A_{2} e^{i\left(\lambda \eta T_{1}+\varphi_{2}\right)}+c c+a_{2} e^{i T_{1}},
\end{aligned}
$$

where $a_{1}=\left(q_{1} E_{1} /\left(\lambda^{2}-1\right)\right)$ and $a_{2}=-\left(q_{1} E_{1} /\left((\lambda \eta)^{2}-1\right)\right)$. 
Substitute equations (30a) and (30b) into equations (29a) and (29b), and then, eliminate secular terms and separate real and imaginary parts, as

$$
\begin{aligned}
D_{2} A_{1}= & -\frac{1}{2 \lambda}\left[\lambda \alpha_{4} A_{1}+\lambda^{2}\left(1-q_{2}\right) E_{2} \sin \varphi_{1}\right], \\
A_{1} D_{2} \varphi_{1}= & -\frac{1}{2 \lambda}\left[\alpha_{1} A_{1}^{3}+\alpha_{2} A_{1} A_{2}^{2}+\left(\lambda \alpha_{3}+\sigma_{1}\right) A_{1}\right. \\
& \left.+\lambda^{2}\left(1-q_{2}\right) E_{2} \cos \varphi_{1}\right], \\
D_{2} A_{2}= & -\frac{1}{2} \beta_{4} A_{2}, \\
A_{2} D_{2} \varphi_{2}= & -\frac{1}{2 \lambda \eta} A_{2}\left[\beta_{1} A_{2}^{2}+\beta_{2} A_{1}^{2}+\left(\lambda \beta_{3}+\eta \sigma_{1}\right) \eta\right],
\end{aligned}
$$

where $\quad \alpha_{1}=3 \gamma_{1}\left(p_{1} F_{1}-F_{2}\right)^{3}, \quad \alpha_{2}=6 \gamma_{1}\left(p_{1} F_{1}-F_{2}\right)$ $\left(p_{2} F_{1}-F_{2}\right)^{2}, \quad \alpha_{3}=-q_{1} p_{1} G_{1}-\left(1-q_{2}\right) G_{2}, \quad \alpha_{4}=q_{1} p_{1} C_{1}+$ $\left(1-q_{2}\right) C_{2}, \quad \beta_{1}=3 \gamma_{2}\left(p_{2} F_{1}-F_{2}\right)^{3}, \quad \beta_{2}=6 \gamma_{2}\left(p_{1} F_{1}-F_{2}\right)^{2}$ $\left(p_{2} F_{1}-F_{2}\right), \beta_{3}=q_{1} p_{2} G_{1}-q_{2} G_{2}$, and $\beta_{4}=-q_{1} p_{2} C_{1}+q_{2} C_{2}$.

Equate right sides of equations (31a)-(31d) to zero; we obtain $A_{2}=0$ due to equation (31c). Since $A_{2}$ is the amplitude of the harmonic term with frequency $\Omega_{2}$ at $\omega_{1} \approx\left(\Omega_{1} / \lambda\right), A_{2}=0$ is correct when $\Omega_{2} \gg \Omega_{1}$ (assumption $2)$. Therefore, equations (31a)-(31d) are simplified as

$$
D_{2} A_{1}=-\frac{1}{2 \lambda}\left[\lambda \alpha_{4} A_{1}+\lambda^{2}\left(1-q_{2}\right) E_{2} \sin \varphi_{1}\right]
$$

$A_{1} D_{2} \varphi_{1}=-\frac{1}{2 \lambda}\left[\alpha_{1} A_{1}^{3}+\left(\lambda \alpha_{3}+\sigma_{1}\right) A_{1}+\lambda^{2}\left(1-q_{2}\right) E_{2} \cos \varphi_{1}\right]$.

Finally, eliminate $\varphi_{1}$ in equations (32a) and (32b); the amplitude frequency equation for the first primary resonance at $\omega_{1} \approx\left(\Omega_{1} / \lambda\right)$ is

$$
\begin{aligned}
& \alpha_{1}^{2} A^{3}+2 \alpha_{1}\left(\lambda \alpha_{3}+\sigma_{1}\right) A^{2}+\left[\left(\lambda \alpha_{3}+\sigma_{1}\right)^{2}+\lambda^{2} \alpha_{4}^{2}\right] A \\
& -\lambda^{4}\left(1-q_{2}\right)^{2} E_{2}^{2}=0,
\end{aligned}
$$

where $A=A_{1}^{2}$.

3.3. Second Primary Resonance at $\omega_{1} \approx \Omega_{1}$. Time scales $T_{1}=$ $\tau$ and $T_{2}=\varepsilon \tau$, and the tuning parameter $\sigma_{2}$ satisfies

$$
\frac{\Omega_{1}^{2}}{\omega_{1}^{2}}=1-\varepsilon \sigma_{2} .
$$

Assume that solutions of equations (25a) and (25b) are

$$
\begin{aligned}
& R_{1}=R_{11}+\varepsilon R_{12}, \\
& R_{2}=R_{21}+\varepsilon R_{22} .
\end{aligned}
$$

Substitute equations (34)-(35b) into equations (25a) and (25b) and equate coefficients of $\varepsilon^{0}$ and $\varepsilon^{1}$ to zero, as $\varepsilon^{0}$ is

$$
\begin{aligned}
D_{1}^{2} R_{11}+R_{11} & =\left(1-q_{2}\right) \lambda^{2} E_{2} e^{i \lambda T_{1}}, \\
D_{1}^{2} R_{21}+\eta^{2} R_{21} & =q_{2} \lambda^{2} E_{2} e^{i \lambda T_{1}} .
\end{aligned}
$$

Also $\varepsilon^{1}$ is

$$
\begin{aligned}
D_{1}^{2} R_{12}+R_{12}= & -2 D_{1} D_{2} R_{11}+\sigma_{2} R_{11}+q_{1} E_{1} e^{i T_{1}} \\
& -q_{1}\left(C_{1}-i G_{1}\right)\left(p_{1} D_{1} R_{11}+p_{2} D_{1} R_{21}\right) \\
& -\left(1-q_{2}\right)\left(C_{2}-i G_{2}\right)\left(D_{1} R_{11}+D_{1} R_{21}\right) \\
& +\gamma_{1}\left[\left(p_{1} F_{1}-F_{2}\right) \bar{R}_{11}+\left(p_{2} F_{1}-F_{2}\right) \bar{R}_{21}\right]^{3} \\
D_{1}^{2} R_{22}+\eta^{2} R_{22}= & -2 D_{1} D_{2} R_{21}+\sigma_{2} \eta^{2} R_{21}-q_{1} E_{1} e^{i T_{1}} \\
& +q_{1}\left(C_{1}-i G_{1}\right)\left(p_{1} D_{1} R_{11}+p_{2} D_{1} R_{21}\right) \\
& -q_{2}\left(C_{2}-i G_{2}\right)\left(D_{1} R_{11}+D_{1} R_{21}\right) \\
& +\gamma_{2}\left[\left(p_{1} F_{1}-F_{2}\right) \bar{R}_{11}+\left(p_{2} F_{1}-F_{2}\right) \bar{R}_{21}\right]^{3} .
\end{aligned}
$$

Assume that general solutions of equations (36a) and (36b) are

$$
\begin{aligned}
& R_{11}=B_{1} e^{i\left(T_{1}+\varphi_{1}\right)}+c c+b_{1} e^{i \lambda T_{1}}, \\
& R_{21}=B_{2} e^{i\left(\eta T_{1}+\varphi_{2}\right)}+c c+b_{2} e^{i \lambda T_{1}}
\end{aligned}
$$

where $b_{1}=\left(\left(1-q_{2}\right) \lambda^{2} E_{2} /\left(1-\lambda^{2}\right)\right)$ and $b_{2}=\left(q_{2} \lambda^{2} E_{2} /\right.$ $\left.\left(\eta^{2}-\lambda^{2}\right)\right)$

Substitute equations (38a) and (38b) into equations (37a) and (37b), and then, eliminate secular terms and separate real and imaginary parts, as

$$
\begin{aligned}
D_{2} B_{1} & =-\frac{1}{2}\left(\alpha_{4} B_{1}+q_{1} E_{1} \sin \varphi_{2}\right), \\
B_{1} D_{2} \varphi_{1} & =-\frac{1}{2}\left[\alpha_{1} B_{1}^{3}+\alpha_{2} B_{1} B_{2}^{2}+\left(\alpha_{3}+\sigma_{2}\right) B_{1}+q_{1} E_{1} \cos \varphi_{2}\right]
\end{aligned}
$$

$$
D_{2} B_{2}=-\frac{1}{2} \beta_{4} B_{2}
$$

$B_{2} D_{2} \varphi_{2}=-\frac{1}{2 \eta} B_{2}\left[\beta_{1} B_{2}^{2}+\beta_{2} B_{1}^{2}+\left(\beta_{3}+\eta \sigma_{2}\right) \eta\right]$.

Equate right sides of equations (39a)-(39d) to zero; we obtain $B_{2}=0$ due to equation (39c). Since $B_{2}$ is the amplitude of the harmonic term with frequency $\Omega_{2}$ at $\omega_{1} \approx \Omega_{1}, B_{2}=0$ is correct when $\Omega_{2} \gg \Omega_{1}$ (assumption 2$)$. Therefore, equations (39a)-(39d) are simplified as

$$
D_{2} B_{1}=-\frac{1}{2}\left(\alpha_{4} B_{1}+q_{1} E_{1} \sin \varphi_{2}\right)
$$

$$
B_{1} D_{2} \varphi_{2}=-\frac{1}{2}\left[\alpha_{1} B_{1}^{3}+\left(\alpha_{3}+\sigma_{2}\right) B_{1}+q_{1} E_{1} \cos \varphi_{2}\right]
$$

Finally eliminating $\varphi_{2}$ in equations (40a) and (40b), the amplitude frequency equation for the second primary resonance at $\omega_{1} \approx \Omega_{1}$ is 


$$
\alpha_{1}^{2} B^{3}+2 \alpha_{1}\left(\alpha_{3}+\sigma_{2}\right) B^{2}+\left[\left(\alpha_{3}+\sigma_{2}\right)^{2}+\alpha_{4}^{2}\right] B-E_{1}^{2} q_{1}^{2}=0
$$

where $B=B_{1}^{2}$.

3.4. Stability Analysis. The Floquet theory [29] is applied to confirm the stability of results solved by the multiple scales method, and the Floquet index can be figured out according to Hsu's method $[30,31]$.

Let $\mathbf{X}=\left[R_{1}, R_{2}, R_{1}^{\prime}, R_{2}^{\prime}\right]^{T}=\left[X_{1}, X_{2}, X_{3}, X_{4}\right]^{T}$; transform equations (25a) and (25b) into $\mathbf{X}^{\prime}(\tau)=\mathbf{F}(\mathbf{X}(\tau), \tau)$, and $\mathbf{X}^{*}$ denotes equilibriums of equations (25a) and (25b). Take a perturbation $\Delta \mathbf{X}$ to $\mathbf{X}^{*}$, as

$$
\Delta \mathbf{X}^{\prime} \approx \frac{\partial \mathbf{F}\left(\tau, \mathbf{X}^{*}\right)}{\partial \mathbf{X}^{*}} \Delta \mathbf{X}=\mathbf{A}\left(\tau, \mathbf{X}^{*}(\tau)\right) \Delta \mathbf{X}
$$

The stability of $\mathbf{X}^{*}$ can be confirmed according to the Jacobi matrix $\mathbf{A}\left(\tau, \mathbf{X}^{*}(\tau)\right)$.

The approximate monodromy matrix can be obtained according to Hsu's method, as

$$
\mathbf{M}=\boldsymbol{\Psi}(T)=\prod_{n=1}^{N_{n}} e^{\mathbf{A}_{n} \Delta T}=\prod_{n=1}^{N_{n}}\left(\mathbf{I}+\sum_{j=1}^{N_{j}} \frac{\left(\mathbf{A}_{n} \Delta T\right)^{j}}{j !}\right),
$$

where I represents the identity matrix, $T$ denotes the time period of the solution, which is divided into $N_{n}$ subintervals, $\Delta T$ is the length of one subinterval, $\mathbf{A}_{n}$ is a constant matrix which represents the time-varying matrix $\mathbf{A}\left(\tau, \mathbf{X}^{*}(\tau)\right)$, and $N_{j}$ represents the number of terms in the approximation of the constant matrix $\mathbf{A}_{n}$ exponential. The Floquet index of matrix $\mathbf{M}$ is available by calculating eigenvalues, and the stability of $\mathbf{X}^{*}$ can be confirmed.

3.5. Numerical Verification. In order to verify the validity of the simplified method for dynamic equations and the amplitude frequency equations solved by the multiple scales method, the multiple scales solutions of the simplified 2DOF equations (22a) and (22b) are compared with Runge-Kutta solutions of original 4DOF equations (17a) and (17b) around the first-order critical speed as shown in Figure 3. The ode45 function in MATLAB (Runge-Kutta method) is applied to solve original $4 \mathrm{DOF}$ equations (17a) and (17b). The initial states of motion for the first rotation speed $\omega_{1}=600 \mathrm{rad} / \mathrm{s}$ are set as $\left[\begin{array}{lllllllll}X_{1} & X_{1}^{\prime} & Y_{1} & Y_{1}^{\prime} & X_{2} & X_{2}^{\prime} & Y_{2} & Y_{2}^{\prime}\end{array}\right]=$ $\left[\begin{array}{llllllll}0 & 0 & 0 & 0 & 0 & 0 & 0 & 0\end{array}\right]$, but for other rotation speeds, the initial states of motion are set as the final states of motion for the previous rotation speed. Integration duration should be long enough to ensure steady-state responses; for equations (17a) and (17b), integration duration are set as $\tau=[0,2000 \pi]$. The root mean square (RMS) [32] is applied to represent the amplitude of the vibration solved by the Runge-Kutta method. The amplitudes of LP and HP rotor are $r_{j}=\sqrt{\left(\sum_{i=1}^{\widehat{N}}\left(X_{j}(i)-\bar{X}_{j}\right)^{2}+\left(Y_{j}(i)-\bar{Y}_{j}\right)^{2} / N\right)}, j=1,2$, where $j$ is the number of points in one period and $\bar{X}_{j}, \bar{Y}_{j}$ are the average values of corresponding response.
In Figure 3, it can be seen that the amplitude frequency curve of the LP rotor is basically the same as that of the HP rotor in appearance, but the amplitude of the LP rotor is much greater than that of the HP rotor, which is because the LP rotor is much more slender than the HP rotor. There are two primary resonances A and B caused by double frequency excitations, which are controlled by amplitude frequency equations equations (33) and (41). Furthermore, the multiple scales solutions almost coincide with the Runge-Kutta solutions, which verify the validity of the simplified method for dynamic equations proposed in this paper and provide a theoretical fundament for the multiple scales method or other analytical methods to solve the nonlinear dynamic equations of the dual-rotor system. The Runge-Kutta solutions are slightly larger than the multiple scales solutions, because dynamic responses solved by the multiple scales method only contain one frequency excitation which plays the most important role while others are ignored. Moreover, the unstable solutions can be attained by this method, while these cannot be attained by the Runge-Kutta method. It can save a lot of calculation time and cost. The results solved by this method can well reveal the inherent mechanism of some unexpected nonlinear phenomena, such as the vibration jump and the resonance hysteresis. All in all, the validity of the simplified method for dynamic equations and the amplitude frequency equations solved by the multiple scales method has been confirmed, based on which the bifurcation analysis can be carried out afterwards.

\section{Bifurcation Analysis}

The bifurcation characteristics for two interrelated primary resonances caused by double frequency excitations of the dual-rotor system are investigated in this section. Nevertheless, the local bifurcation characteristics for two primary resonances mostly depend on dynamic parameters of the dual-rotor and intershaft bearing systems, in which the linear stiffness and nonlinear stiffness of the intershaft bearing play the most important roles. Therefore, this section focuses on the effect of the intershaft bearing's linear stiffness and nonlinear stiffness on the bifurcation characteristics for two interrelated primary resonances. Considering the complexity of the amplitude frequency equations, a two-state-variable singularity method $[21,22,33]$ is applied to describe bifurcation modes for two primary resonances of the dual-rotor system in an engineering way. Amplitude frequency equations equations (33) and (41) are directly taken as the engineering unfolding, instead of the universal unfolding. The engineering unfolding may not be logical in mathematics, but it is of very important significance for engineering.

Substitute $\sigma_{1}=\lambda^{2}-\sigma$ into equation (33) and $\sigma_{2}=1-\sigma$ into equation (41), and take $\sigma=\left(\Omega_{1}^{2} / \omega_{1}^{2}\right)$ as the bifurcation parameter and $\alpha_{1}$ and $\alpha_{4}$ as the unfolding parameters; the left side of equations (33) and (41) could be viewed as the engineering unfolding for two primary resonances of the dualrotor system, as 


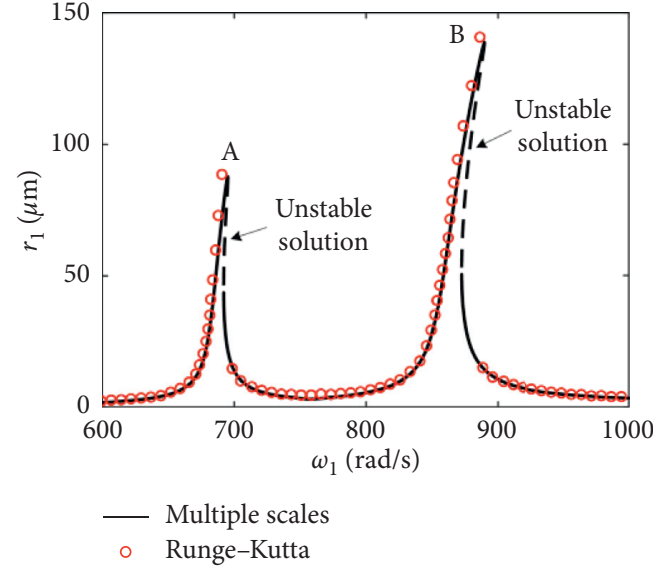

(a)

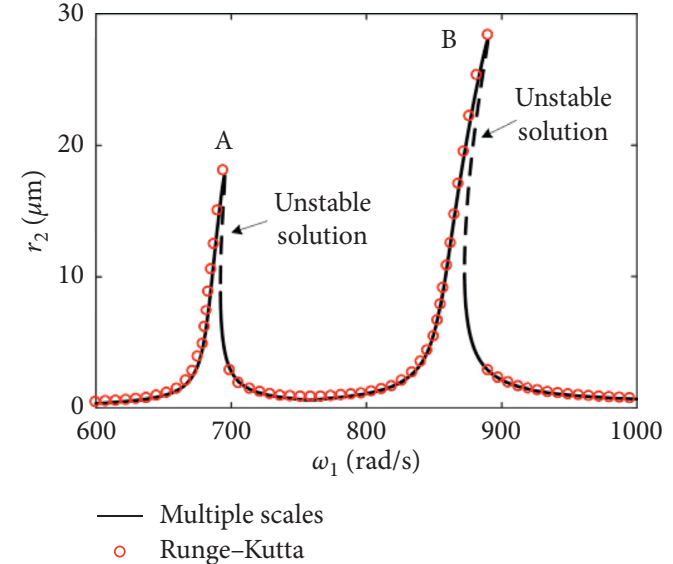

(b)

Figure 3: Amplitude frequency curves solved by the multiple scales method and the Runge-Kutta method. (a) For the LP rotor. (b) For the HP rotor. Solid lines represent stable solutions, and dotted lines represent unstable solutions.

$$
\begin{aligned}
\mathbf{F}_{1}= & \alpha_{1}^{2} A^{3}+2 \alpha_{1}\left(\lambda \alpha_{3}+\lambda^{2}-\sigma\right) A^{2} \\
& +\left[\left(\lambda \alpha_{3}+\lambda^{2}-\sigma\right)^{2}+\lambda^{2} \alpha_{4}^{2}\right] A-\lambda^{4}\left(1-q_{2}\right)^{2} E_{2}^{2} \\
\mathbf{F}_{2}= & \alpha_{1}^{2} B^{3}+2 \alpha_{1}\left(\alpha_{3}+1-\sigma\right) B^{2} \\
& +\left[\left(\alpha_{3}+1-\sigma\right)^{2}+\alpha_{4}^{2}\right] B-E_{1}^{2} q_{1}^{2} .
\end{aligned}
$$

The derivatives of $\mathbf{F}_{1}$ and $\mathbf{F}_{2}$ can be expressed as

$$
\mathbf{F}_{1 A}=3 \alpha_{1}^{2} A^{2}+4 \alpha_{1}\left(\lambda \alpha_{3}+\lambda^{2}-\sigma\right) A+\left(\lambda \alpha_{3}+\lambda^{2}-\sigma\right)^{2}+\lambda^{2} \alpha_{4}^{2},
$$

$\mathbf{F}_{1 A A}=6 \alpha_{1}^{2} A+4 \alpha_{1}\left(\lambda \alpha_{3}+\lambda^{2}-\sigma\right)$

$$
\mathbf{F}_{1 \sigma}=-2 \alpha_{1} A^{2}-2\left(\lambda \alpha_{3}+\lambda^{2}-\sigma\right) A,
$$

$\mathbf{F}_{2 B}=3 \alpha_{1}^{2} B^{2}+4 \alpha_{1}\left(\alpha_{3}+1-\sigma\right) B+\left(\alpha_{3}+1-\sigma\right)^{2}+\alpha_{4}^{2}$,

$\mathbf{F}_{2 B B}=6 \alpha_{1}^{2} B+4 \alpha_{1}\left(\alpha_{3}+1-\sigma\right)$,

$\mathbf{F}_{2 \sigma}=-2 \alpha_{1} B^{2}-2\left(\alpha_{3}+1-\sigma\right) B$,

$$
\mathbf{F}_{1 B}=\mathbf{F}_{2 A}=\mathbf{F}_{1 A B}=\mathbf{F}_{2 A B}=0 \text {. }
$$

Transition sets are consisted of three parts, which are bifurcation set, hysteresis set, and double limit set. The bifurcation set indicates that unfolding equations (44a) and (44b) contain a singular point. The hysteresis set indicates that the unfolding makes at least quadratic contact with the vertical plane of a constant bifurcation parameter. The double limit set indicates that the unfolding exists for two different limit points for the same bifurcation parameter [21]. According to the two-variable singularity method, transition sets for the bifurcation equations can be expressed as follows:

Bifurcation set is as follows:

$$
\operatorname{Bi}=\left\{\left(\alpha_{1}, \alpha_{4}\right) \in R^{2} \mid \begin{array}{c}
\exists(A, B, \sigma) \text { s.t. } \mathbf{F}_{1}=0, \mathbf{F}_{2}=0, \\
\mathbf{F}_{1 A} \mathbf{F}_{2 B}-\mathbf{F}_{1 B} \mathbf{F}_{2 A}=0, \\
\mathbf{F}_{1 A} \mathbf{F}_{2 \sigma}-\mathbf{F}_{1 \sigma} \mathbf{F}_{2 A}=0
\end{array}\right\} .
$$

Hysteresis set is as follows:

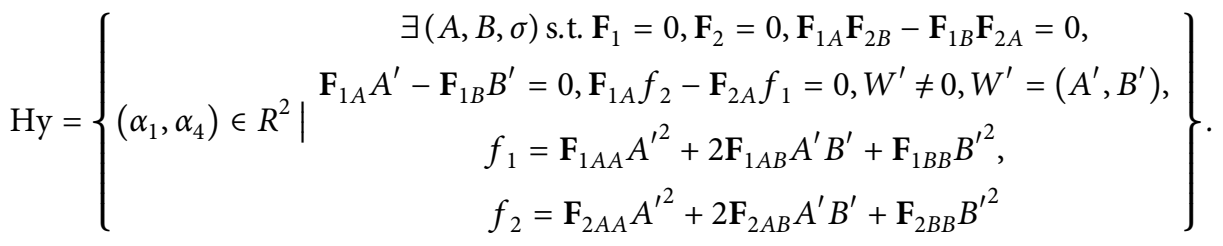

Double limit set is as follows: 


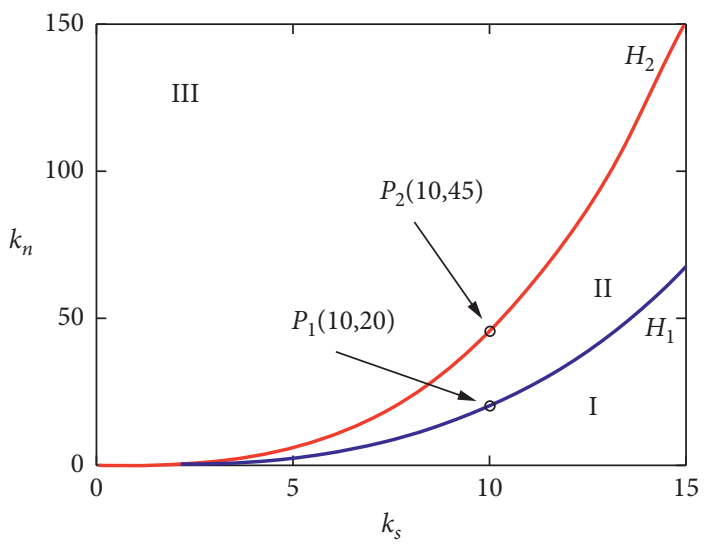

FIgURE 4: Transition sets in $k_{n}-k_{s}$ parameters plane.

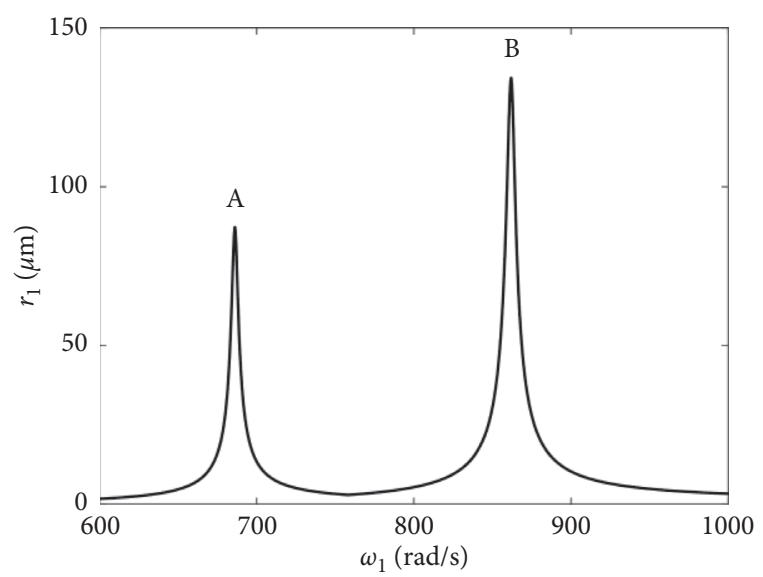

(a)

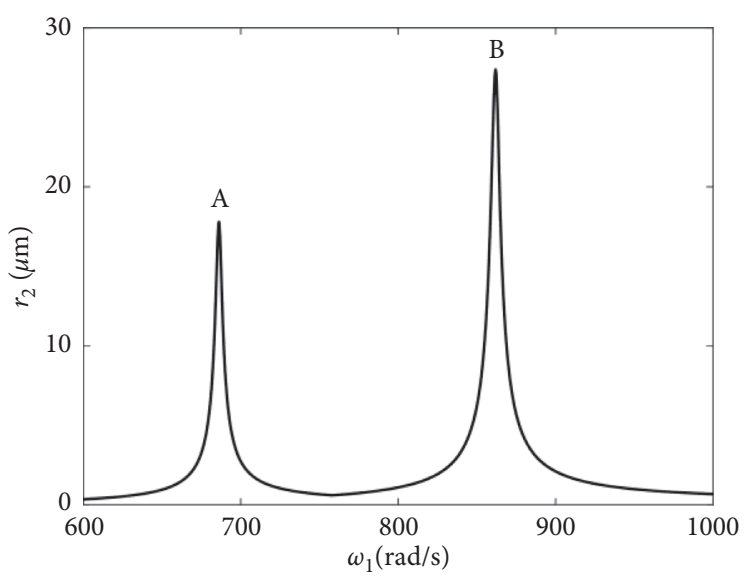

(b)

Figure 5: Amplitude frequency curves for the bifurcation mode of the region I in Figure $4\left(k_{n}=1 \times 10^{23} \mathrm{~N} / \mathrm{m}^{3}, k_{s}=12 \times 10^{8} \mathrm{~N} / \mathrm{m}\right)$. (a) For the LP rotor. (b) For the HP rotor. Solid lines represent stable solutions, and dotted lines represent unstable solutions.

$$
\mathrm{DL}=\left\{\left(\alpha_{1}, \alpha_{4}\right) \in R^{2} \mid \begin{array}{c}
\exists\left(W_{1}, W_{2}, \sigma\right) \text { s.t. } \mathbf{F}_{1}=0, \mathbf{F}_{2}=0, \\
\\
\mathbf{F}_{1 A} \mathbf{F}_{2 B}-\mathbf{F}_{1 B} \mathbf{F}_{2 A}=0, \\
W_{1} \neq W_{2}, W=(A, B)
\end{array}\right\} .
$$

According to above calculation procedure equations (44a)-(46c), the transition sets for two primary resonances of the dual-rotor system can be attained. The transition sets contain two hysteresis set curves $H_{1}$ and $H_{2}$, as shown in the $k_{n}-k_{s}$ parameters plane in Figure 4 with $k_{s}$ from 0 to 15 $\left(10^{8} \mathrm{~N} / \mathrm{m}\right)$ and $k_{n}$ from 0 to $150\left(10^{23} \mathrm{~N} / \mathrm{m}^{3}\right)$. There are three regions divided by $H_{1}$ and $H_{2}$ in the $k_{n}-k_{s}$ parameters plane, which indicates that two primary resonances of the dual-rotor system have three different bifurcation modes. Three different bifurcation modes for three regions I, II, and III can be obtained, as shown in Figures 5-7. Moreover, two critical bifurcation modes for two hysteresis set curves $H_{1}$ and $H_{2}$ can also be obtained, as shown in Figures 8 and 9.

Figure 5 shows the bifurcation mode of region I in Figure 4 , in which the linear stiffness $k_{s}$ is rather large and the nonlinear stiffness $k_{n}$ is rather small. It can found that two primary resonances A and B have a linear characteristics. The dynamic characteristics of the dual-rotor system are almost the same with the linear system in this case. Figure 6 shows the bifurcation mode of region II in Figure 4, in which the linear stiffness is slightly smaller or the nonlinear stiffness is slightly larger. It can be found that the second primary resonance $B$ shows vibration jump and resonance hysteresis while the first primary resonance A does not, which means $B$ shows the hardening characteristics while A does not. This phenomenon indicates that $B$ is more prone to show nonlinear phenomena than A. Figure 7 shows the bifurcation mode of region III in Figure 4, in which the linear stiffness is rather small and the nonlinear stiffness is rather large. It can be found that both primary resonances $\mathrm{A}$ and B show vibration jump and resonance hysteresis, which indicates that both $\mathrm{A}$ and $\mathrm{B}$ show the hardening characteristics. The dynamic characteristics of the dual-rotor system have essential differences with the linear system in this case.

Furthermore, two critical bifurcation modes for two hysteresis set curves $H_{1}$ and $H_{2}$ are investigated. Figure 8 shows the bifurcation mode of hysteresis set curve $H_{1}$, in 


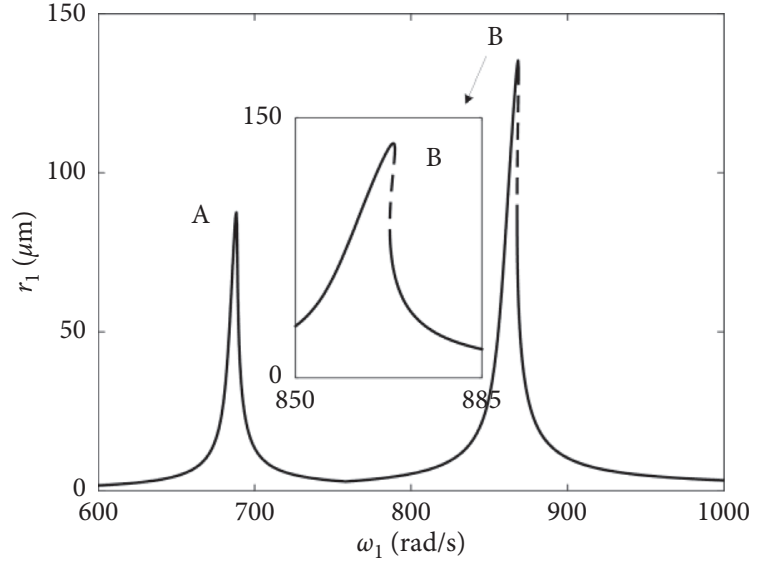

(a)

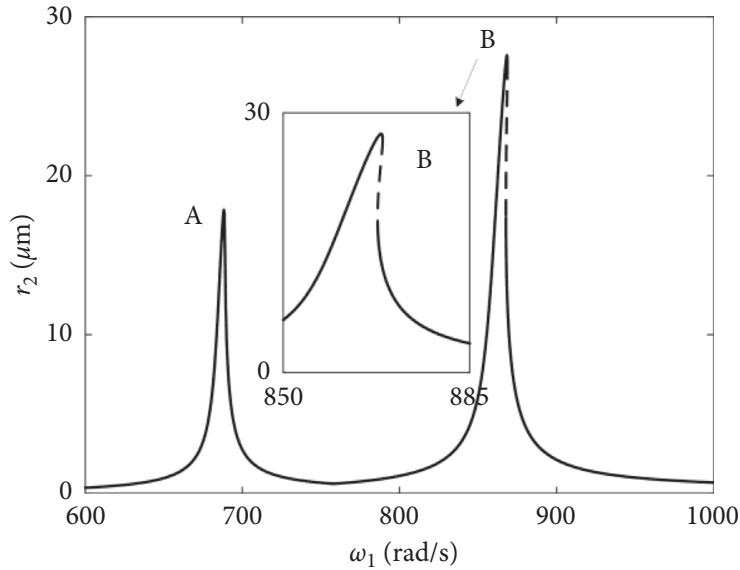

(b)

Figure 6: Amplitude frequency curves for the bifurcation mode of the region II in Figure $4\left(k_{n}=30 \times 10^{23} \mathrm{~N} / \mathrm{m}^{3}, k_{s}=9 \times 10^{8} \mathrm{~N} / \mathrm{m}\right)$. (a) For the LP rotor. (b) For the HP rotor. Solid lines represent stable solutions, and dotted lines represent unstable solutions.

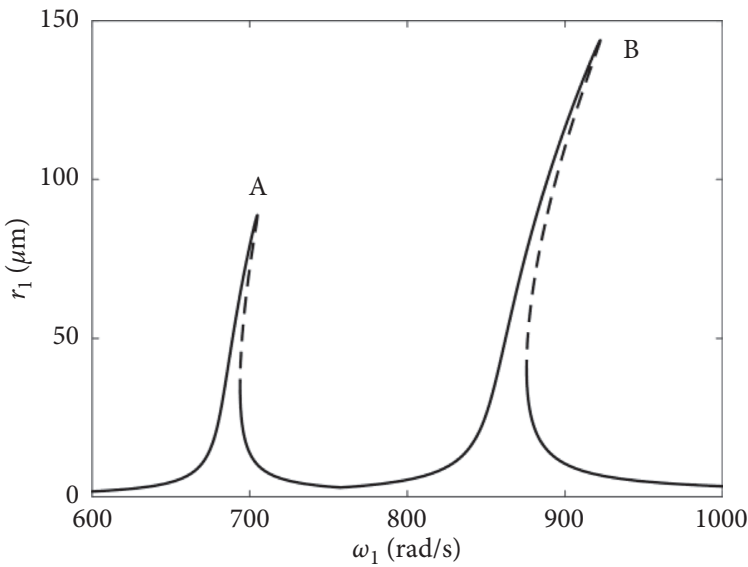

(a)

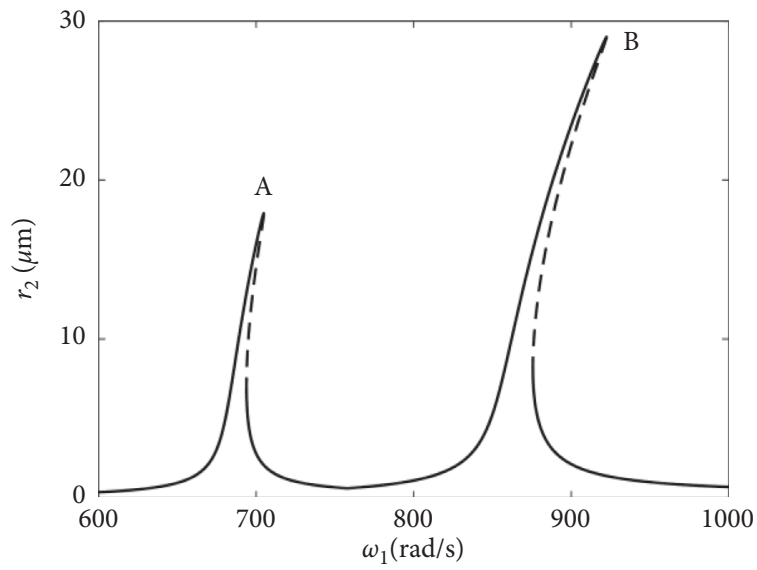

(b)

Figure 7: Amplitude frequency curves for the bifurcation mode of the region III in Figure $4\left(k_{n}=80 \times 10^{23} \mathrm{~N} / \mathrm{m}^{3}, k_{s}=8 \times 10^{8} \mathrm{~N} / \mathrm{m}\right)$. (a) For the LP rotor. (b) For the HP rotor. Solid lines represent stable solutions, and dotted lines represent unstable solutions.

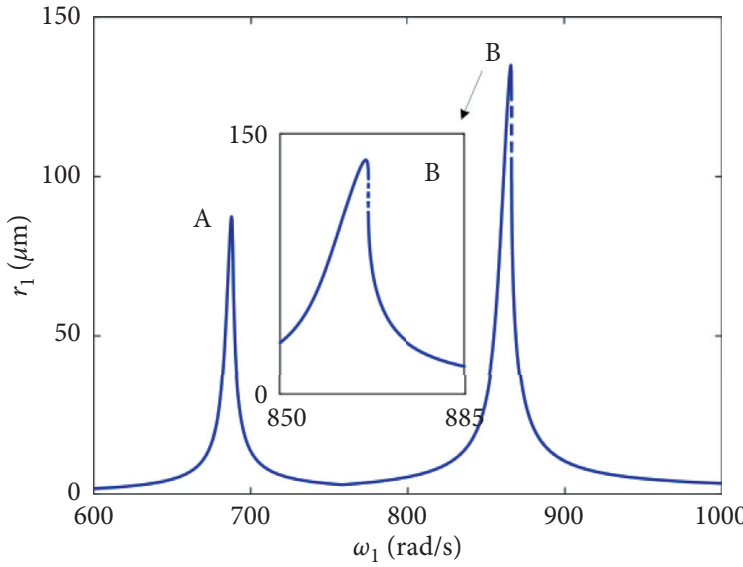

(a)

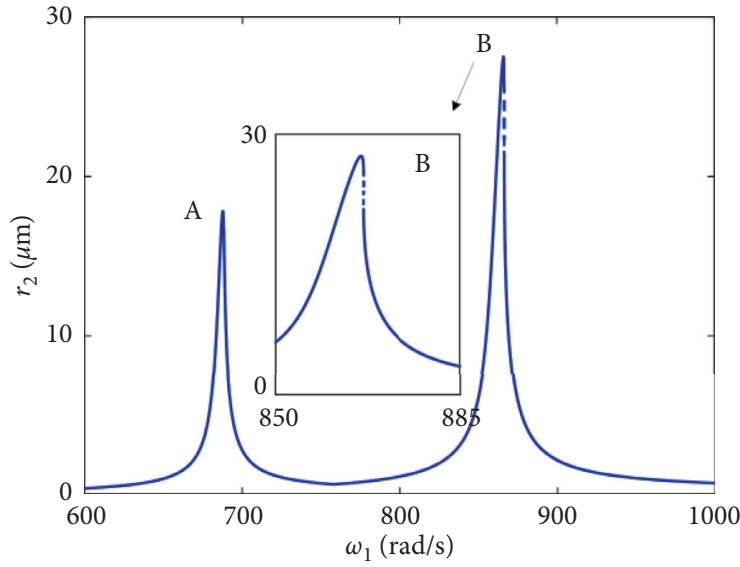

(b)

Figure 8: Amplitude frequency curves for the bifurcation mode of the hysteresis set curve $H_{1}$ in Figure 4 $\left(k_{n}=20 \times 10^{23} \mathrm{~N} / \mathrm{m}^{3}, k_{s}=10 \times 10^{8} \mathrm{~N} / \mathrm{m}\right)$. (a) For the LP rotor. (b) For the HP rotor. Solid lines represent stable solutions, and dotted lines represent unstable solutions. 


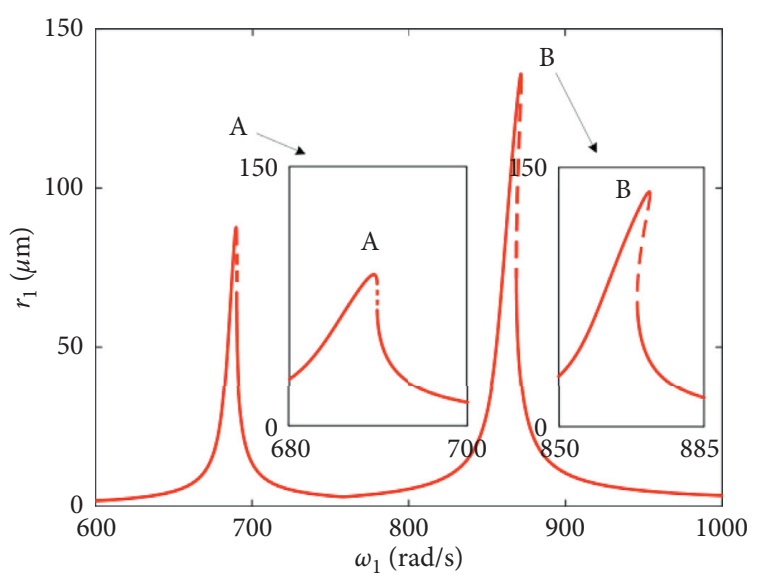

(a)

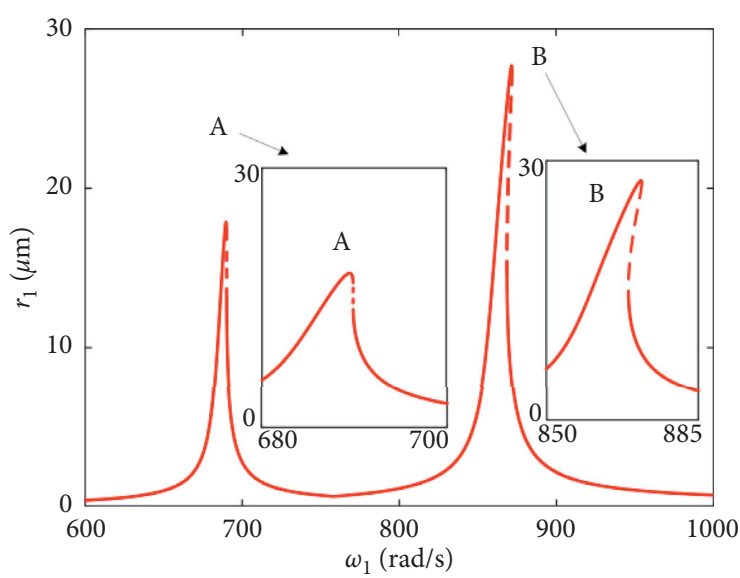

(b)

FIgure 9: Amplitude frequency curves for the bifurcation mode of the hysteresis set curve $H_{2}$ in Figure 4 $\left(k_{n}=45 \times 10^{23} \mathrm{~N} / \mathrm{m}^{3}, k_{s}=10 \times 10^{8} \mathrm{~N} / \mathrm{m}\right)$. (a) For the LP rotor. (b) For the HP rotor. Solid lines represent stable solutions, and dotted lines represent unstable solutions.

which the second primary resonance $B$ just begins to show vibration jump in this case, while the first primary resonance A has linear characteristics. Figure 9 shows the bifurcation mode of hysteresis set curve $\mathrm{H}_{2}$, in which the first primary resonance $A$ just begins to show vibration jump in this case, while the second primary resonance $\mathrm{B}$ has nonlinear characteristics.

\section{Conclusions}

In this paper, a simplified method for dynamic equations under double frequency excitations of a simple dual-rotor system considering the nonlinearity of the intershaft bearing has been proposed, based on which bifurcation modes for two interrelated primary resonances caused by double frequency excitations have been investigated theoretically. The multiple scales method has been applied to calculate amplitude frequency equations for two interrelated primary resonances, which have been taken as the engineering unfolding directly to investigate bifurcation modes of the dual-rotor system based on the two-state-variable singularity method. The results show that the linear stiffness and the nonlinear stiffness of the intershaft bearing have a significant influence on bifurcation modes of the system. The transition set, including two hysteresis set curves, divides the parameters plane $k_{n}-k_{s}$ into three regions, indicating that there exists three different types of bifurcation modes for two interrelated primary resonances, which are, respectively, for both primary resonances having linear characteristics and for second primary resonance having hardening characteristics while first primary resonance having linear characteristics and for both primary resonances having hardening characteristics. The future work will concentrate on the experimental study of the dual-rotor system.

\section{Appendix}

The nonlinear force of the intershaft bearing in the complexnumber coordinate is

$$
\begin{aligned}
F_{b}= & \frac{F_{x}}{e_{1}}+i \frac{F_{y}}{e_{1}} \\
= & k_{s}\left(X_{i}-X_{o}\right)+e_{1}^{2} k_{n}\left(X_{i}-X_{o}\right)\left[\left(X_{i}-X_{o}\right)^{2}\right. \\
& \left.-3\left(Y_{i}-Y_{o}\right)^{2}\right]+i\left\{k_{s}\left(Y_{i}-Y_{o}\right)+e_{1}^{2} k_{n}\left(Y_{i}-Y_{o}\right)\right. \\
& \left.\cdot\left[\left(Y_{i}-Y_{o}\right)^{2}-3\left(X_{i}-X_{o}\right)^{2}\right]\right\} \\
= & k_{s}\left(r_{i}-r_{o}\right)+e_{1}^{2} k_{n}\left\{( X _ { i } - X _ { o } ) \left[\left(X_{i}-X_{o}\right)^{2}\right.\right. \\
& \left.\left.-3\left(Y_{i}-Y_{o}\right)^{2}\right]+i\left(Y_{i}-Y_{o}\right)\left[\left(Y_{i}-Y_{o}\right)^{2}-3\left(X_{i}-X_{o}\right)^{2}\right]\right\},
\end{aligned}
$$

where the brace $\left\{\left(X_{i}-X_{o}\right) \quad\left[\left(X_{i}-X_{o}\right)^{2}-3\left(Y_{i}-Y_{o}\right)^{2}\right]+\right.$ $\left.i\left(Y_{i}-Y_{o}\right)\left[\left(Y_{i}-Y_{o}\right)^{2}-3\left(X_{i}-X_{o}\right)^{2}\right]\right\}$ is formulated as 


$$
\begin{aligned}
\{\}= & \left(X_{i}^{3}-X_{o}^{3}-3 X_{i}^{2} X_{o}+3 X_{i} X_{o}^{2}\right)-3\left(X_{i} Y_{i}^{2}-X_{o} Y_{o}^{2}-X_{o} Y_{i}^{2}-2 X_{i} Y_{i} Y_{o}+X_{i} Y_{o}^{2}+2 X_{o} Y_{i} Y_{o}\right)+i\left(Y_{i}^{3}-Y_{o}^{3}-3 Y_{i}^{2} Y_{o}+3 Y_{i} Y_{o}^{2}\right) \\
& -3 i\left(X_{i}^{2} Y_{i}-X_{o}^{2} Y_{o}-X_{i}^{2} Y_{o}-2 X_{i} X_{o} Y_{i}+X_{o}^{2} Y_{i}+2 X_{i} X_{o} Y_{o}\right) \\
= & \left(X_{i}^{3}+i Y_{i}^{3}-3 X_{i} Y_{i}^{2}-3 i X_{i}^{2} Y\right)-\left(X_{o}^{3}+i Y_{o}^{3}-3 X_{o} Y_{o}^{2}-3 i X_{o}^{2} Y_{o}\right)-3\left(X_{i}^{2} X_{o}+i Y_{i}^{2} Y_{o}-X_{o} Y_{i}^{2}-i X_{i}^{2} Y_{o}-2 X_{i} Y_{i} Y_{o}-2 i X_{i} X_{o} Y_{i}\right) \\
& +3\left(X_{i} X_{o}^{2}+i Y_{i} Y_{o}^{2}-X_{i} Y_{o}^{2}-i X_{o}^{2} Y_{i}-2 X_{o} Y_{i} Y_{o}-2 i X_{i} X_{o} Y_{o}\right) \\
= & \left(X_{i}-i Y_{i}\right)^{3}-\left(X_{o}-i Y_{o}\right)^{3}-3\left(X_{i}-i Y_{i}\right)^{2}\left(X_{o}-i Y_{o}\right)+3\left(X_{i}-i Y_{i}\right)\left(X_{o}-i Y_{o}\right)^{2} \\
= & \bar{r}_{i}^{3}-\bar{r}_{o}^{3}-3 \bar{r}_{i}^{2} \bar{r}_{o}+3 \bar{r}_{i} \bar{r}_{o}^{2} \\
= & \left(\bar{r}_{i}-\bar{r}_{o}\right)^{3} .
\end{aligned}
$$

Thus, the nonlinear force of the intershaft bearing in the complex-number coordinate is

$$
\begin{aligned}
F_{b} & =F_{X}+i F_{Y}=k_{s}\left(r_{i}-r_{o}\right)+e_{1}^{2} k_{n}\left(\bar{r}_{i}-\bar{r}_{o}\right)^{3} \\
& =k_{s}\left(F_{1} r_{1}-F_{2} r_{2}\right)+e_{1}^{2} k_{n}\left(F_{1} \bar{r}_{1}-F_{2} \bar{r}_{2}\right)^{3} .
\end{aligned}
$$

\section{Data Availability}

The dynamic simulation data used to support the findings of this study are included within the article.

\section{Conflicts of Interest}

The authors declare that they have no conflicts of interest.

\section{Acknowledgments}

The authors are very grateful for the financial supports from the National Major Science and Technology Projects of China (Grant No. 2017-IV-0008-0045) and the National Natural Science Foundation of China (Grant No. 11972129).

\section{References}

[1] Y. S. Chen and H. B. Zhang, "Review and prospect on the research of dynamics of complete aero-engine systems," Acta Aeronautica Et Astronautica Sinica, vol. 32, no. 8, pp. 13711391, 2011.

[2] Q. Li, L. Yan, and J. F. Hamilton, "Investigation of the steadystate response of a dual-rotor system with intershaft squeeze film damper," Journal of Engineering for Gas Turbines and Power, vol. 108, no. 4, pp. 605-612, 1986.

[3] Z. Ji and J. W. Zu, "Method of multiple scales for vibration analysis of rotor shaft systems with non-linear bearing pedestal model," Journal of Sound and Vibration, vol. 218, no. 2, pp. 293-305, 1998.

[4] K. Kappaganthu and C. Nataraj, "Nonlinear modeling and analysis of a rolling element bearing with a clearance," Communications in Nonlinear Science and Numerical Simulation, vol. 16, no. 10, pp. 4134-4145, 2011.

[5] L. J. López-Reyes and E. V. Kurmyshev, "Parametric resonance in nonlinear vibrations of string under harmonic heating," Communications in Nonlinear Science and $\mathrm{Nu}$ merical Simulation, vol. 55, pp. 146-156, 2018.
[6] D. H. Hibner, "Dynamic response of viscous-damped multishaft jet engines," Journal of Aircraft, vol. 12, no. 4, pp. 305-312, 1975.

[7] K. Gupta, K. D. Gupta, and K. Athre, "Unbalance response of a dual rotor system: theory and experiment," Journal of Vibration and Acoustics, vol. 115, no. 4, pp. 427-435, 1993.

[8] G. Ferraris, V. Maisonneuve, and M. Lalanne, "Prediction of the dynamic behavior of non-symmetrical coaxial co- or counter-rotating rotors," Journal of Sound and Vibration, vol. 195, no. 4, pp. 649-666, 1996.

[9] M. Guskov, J.-J. Sinou, F. Thouverez, and O. S. Naraikin, "Experimental and numerical investigations of a dual-shaft test rig with intershaft bearing," International Journal of Rotating Machinery, vol. 2007, Article ID 75762, 12 pages, 2007.

[10] Z. X. Zhang, Q. Zhang, X. L. Li, and T. L. Qian, “The wholebeat correlation method for the identification of an unbalance response of a dual-rotor system with a slight rotating speed difference," Mechanical Systems and Signal Processing, vol. 25, no. 5, pp. 1667-1673, 2011.

[11] Z. X. Zhang, L. Z. Wang, Z. J. Jin, Q. Zhang, and X. L. Li, "Non-whole beat correlation method for the identification of an unbalance response of a dual-rotor system with a slight rotating speed difference," Mechanical Systems and Signal Processing, vol. 39, no. 1-2, pp. 452-460, 2013.

[12] Y. Yang, D. Cao, T. Yu, D. Wang, and C. Li, "Prediction of dynamic characteristics of a dual-rotor system with fixed point rubbing-theoretical analysis and experimental study," International Journal of Mechanical Sciences, vol. 115-116, pp. 253-261, 2016.

[13] N. Wang, D. Jiang, and K. Behdinan, "Vibration response analysis of rubbing faults on a dual-rotor bearing system," Archive of Applied Mechanics, vol. 87, no. 11, pp. 1891-1907, 2017.

[14] N. Wang, C. Liu, D. Jiang, and K. Behdinan, "Casing vibration response prediction of dual-rotor-blade-casing system with blade-casing rubbing," Mechanical Systems and Signal Processing, vol. 118, pp. 61-77, 2019.

[15] C. Sun, Y. Chen, and L. Hou, "Steady-state response characteristics of a dual-rotor system induced by rub-impact," Nonlinear Dynamics, vol. 86, no. 1, pp. 91-105, 2016.

[16] C. Sun, Y. Chen, and L. Hou, "Nonlinear dynamical behaviors of a complicated dual-rotor aero-engine with rub-impact," Archive of Applied Mechanics, vol. 88, no. 8, pp. 1305-1324, 2018.

[17] Y. S. Chen, Nonlinear Vibrations, Higher Education Press, Beijing, China, 2002.

[18] M. Golubitsky and D. G. Schaeffer, Singularities and Groups in Bifurcation Theory, Springer, Berlin, Germany, 1985. 
[19] Y. S. Chen and W. Langford, "The subharmonic bifurcation solution of nonlinear mathieus equation and Euler dynamically buckling problem," Acta Mechanica Sinica, vol. 4, pp. 350-362, 1988.

[20] Y. S. Chen and A. Y. Leung, Bifurcation and Chaos in Engineering, Springer, Berlin, Germany, 1998.

[21] Z. Qin and Y. Chen, "Singular analysis of bifurcation systems with two parameters," Acta Mechanica Sinica, vol. 26, no. 3, pp. 501-507, 2010.

[22] Z. Qin, Y. Chen, X. Zhan, B. Liu, and K. Zhu, "Research on the galloping and anti-galloping of the transmission line," International Journal of Bifurcation and Chaos, vol. 22, no. 2, Article ID 1250038, 2012.

[23] P. Gao, L. Hou, R. Yang, and Y. Chen, "Local defect modelling and nonlinear dynamic analysis for the inter-shaft bearing in a dual-rotor system," Applied Mathematical Modelling, vol. 68, pp. 29-47, 2019.

[24] L. Hou, Y. Chen, Y. Fu, H. Chen, Z. Lu, and Z. Liu, “Application of the HB-AFT method to the primary resonance analysis of a dual-rotor system," Nonlinear Dynamics, vol. 88, no. 4, pp. 2531-2551, 2017.

[25] M. K. Al-Solihat and K. Behdinan, "Nonlinear dynamic response and transmissibility of a flexible rotor system mounted on viscoelastic elements," Nonlinear Dynamics, vol. 97, no. 2, pp. 1581-1600, 2019.

[26] N. V. Perepelkin, Y. V. Mikhlin, and C. Pierre, "Non-linear normal forced vibration modes in systems with internal resonance," International Journal of Non-linear Mechanics, vol. 57, pp. 102-115, 2013.

[27] Y. Jin, Z. Lu, R. Yang, L. Hou, and Y. Chen, “A new nonlinear force model to replace the Hertzian contact model in a rigidrotor ball bearing system," Applied Mathematics and Mechanics, vol. 39, no. 3, pp. 365-378, 2018.

[28] H. Chen, L. Hou, and Y. Chen, "Bifurcation analysis of a rigidrotor squeeze film damper system with unsymmetrical stiffness supports," Archive of Applied Mechanics, vol. 87, no. 8, pp. 1347-1364, 2017.

[29] Z. Zhang, Y. Chen, and Q. Cao, "Bifurcations and hysteresis of varying compliance vibrations in the primary parametric resonance for a ball bearing," Journal of Sound and Vibration, vol. 350, pp. 171-184, 2015.

[30] P. Friedmann, C. E. Hammond, and T.-H. Woo, "Efficient numerical treatment of periodic systems with application to stability problems," International Journal for Numerical Methods in Engineering, vol. 11, no. 7, pp. 1117-1136, 1977.

[31] Z.-y. Zhang and Y.-s. Chen, "Harmonic balance method with alternating frequency/time domain technique for nonlinear dynamical system with fractional exponential," Applied Mathematics and Mechanics, vol. 35, no. 4, pp. 423-436, 2014.

[32] N. Tandon and A. Choudhury, "A review of vibration and acoustic measurement methods for the detection of defects in rolling element bearings," Tribology International, vol. 32, no. 8, pp. 469-480, 1999.

[33] L. Hou, Y. Chen, Z. Lu, and Z. Li, "Bifurcation analysis for $2: 1$ and 3:1 super-harmonic resonances of an aircraft cracked rotor system due to maneuver load," Nonlinear Dynamics, vol. 81, no. 1-2, pp. 531-547, 2015. 